\title{
Distribución de equinodermos en el golfo San José y sur del golfo San Matías (Chubut, Argentina)
}

\author{
Echinoderms distribution in the San José and South of the San Matías Gulfs (Chubut, Argentina) \\ Héctor E. Zaixso ${ }^{1}$ y Zulma I. Lizarralde ${ }^{2}$ \\ ${ }^{l}$ Consejo Nacional de Investigaciones Cientificas y Técnicas. Universidad Nacional de la Patagonia S. J. Bosco, \\ Facultad de Ciencias Naturales, Ciudad Universitaria, km 4, 9000 Comodoro Rivadavia, Chubut, Argentina. \\ ${ }^{2}$ Universidad Nacional de la Patagonia Austral. Unidad Académica Rio Gallegos, \\ Lisandro de la Torre 1070, 9400 Río Gallegos, Santa Cruz, Argentina. \\ hzaixso@impsat1.com.ar
}

\begin{abstract}
The distribution of the most frequent species of subtidal echinoderms of the San José Gulf and south of the San Matías Gulf (Chubut Province, Argentina: 42 ${ }^{\circ} 20^{\prime} \mathrm{S}$ and 64 $20^{\prime}$ $\mathrm{W})$ is studied. The selected species were the echinoids Trypilaster philippii, Pseudechinus magellanicus and Arbacia dufresnei; the ophiuroids Ophioplocus januarii, Ophiactis asperula, Amphiura crassipes and Amphiura magellanica and the holothurians Chiridota marenzelleri and Pentamera chiloensis. The densities (individuals $\mathrm{m}^{-2}$ ) of the species were drawn on the Gulf chart using the point kriging method. The environmental parameters recorded in each sampling station were: depth, substrata granulometry, presence of hard substrata, velocity of the surface currents, bottom temperatures in August and December and presence of seaweeds. The data were analyzed by canonical correspondence analysis (CCA) with a forward selection of environmental variables and the complementary use of HOF (Huisman, Olff and Fresco) hierarchical models in the analysis of abundance response curves in ecological gradients. Several groups of species were defined using these methods: 1- A group formed by the echinoids Arbacia dufresnei and Pseudechinus magellanicus and the ophiuroids Ophioplocus januarii, Amphiura magellanica and Ophiactis asperula, which presented a negative monotonic relationship to the increasing quantity of the very fine sand fraction, some of them are positively associated to strong surface currents ( $P$. magellanicus, A. magellanica and $O$. asperula), other to shallow waters (A. dufresnei and $O$. januarii). Ophiactis asperula and Pseudechinus magellanicus are the most abundant echinoderms in these gulfs. The holothurian Pentamera chiloensis, which is near to this first group in the CCA, is not related to any of the analyzed environmental factors. 2- The other species (Trypilaster philippii, Amphiura crassipes and Chiridota marenzelleri), have all of them preference for substrates of very fine granulometry but differ in other habitat characteristics. The possible feeding habits of these species are discussed.
\end{abstract}

Key words: Abundance, environmental factors, CCA, HOF models, feeding habits.
Resumen.- En este trabajo se estudia la distribución de los equinodermos submareales más frecuentes del golfo San José y sur del golfo San Matías (provincia del Chubut, Argentina: $42^{\circ}$ $20^{\prime} \mathrm{S}$ y $64^{\circ} 20^{\prime} \mathrm{W}$ ). Las especies seleccionadas fueron los equinoideos Trypilaster philippii, Pseudechinus magellanicus y Arbacia dufresnei; los ofiuroideos Ophioplocus januarii, Ophiactis asperula, Amphiura crassipes y Amphiura magellanica y los holoturoideos Chiridota marenzelleri y Pentamera chiloensis. Las densidades (individuos $\mathrm{m}^{-2}$ ) de estas especies fueron volcadas sobre sendas cartas del área de estudio utilizando el método de "point kriging". En cada estación de muestreo se obtuvieron los siguientes parámetros ambientales: profundidad, granulometría del sustrato, presencia de sustratos duros, velocidad de las corrientes en superficie, las temperaturas de fondo en agosto y diciembre y la presencia de macroalgas. Los datos fueron analizados mediante un análisis canónico de correspondencias (CCA) con una selección "forward" de variables ambientales y el uso complementario de modelos jerárquicos HOF (Huisman, Olff y Fresco) para el análisis de las curvas respuesta de las abundancias en gradientes ecológicos. Varios grupos de especies fueron definidos con el uso de estos métodos: 1- un grupo formado por los equinoideos Arbacia dufresnei y Pseudechinus magellanicus y los ofiuroideos Ophioplocus januarii, Amphiura magellanica y Ophiactis asperula, que presentan una relación monotónica negativa con el aumento de la cantidad de la fracción de arenas muy finas. Algunas de estas especies están asociadas positivamente con corrientes de superficie fuertes ( $P$. magellanicus, A. magellanica y $O$. asperula), en tanto que otras lo están a aguas someras $(A$. dufresnei y O. januarii). Ophiactis asperula y Pseudechinus magellanicus son los equinodermos más abundantes en el área de muestreo. El holoturoideo Pentamera chiloensis, que se halla cerca del grupo anterior en el CCA, no está relacionado con ninguno de los factores ambientales analizados. 2- Las otras especies (Trypilaster philippii, Amphiura crassipes y Chiridota marenzelleri), muestran todas ellas preferencia por sustratos de granulometría muy fina, pero difieren entre sí en otras características de su hábitat. Se discuten los posibles hábitos alimenticios de estas especies.

Palabras clave: Abundancia, factores ambientales, CCA, modelos HOF, hábitos alimenticios.

\section{Introducción}

En Argentina no existen prácticamente antecedentes de estudios referidos a la ecología de las especies de equinodermos en sus costas. La escasa información existente se halla incluida en trabajos de índole general, entre los cuales se pueden citar Escofet et al. (1978), Penchaszadeh (1979), Carriquiriborde et al. (1983), 
Bastida et al. (1992) y Zaixso et al. (1998).

En el marco de un programa de estudios de las comunidades submareales de los golfos norpatagónicos, se realizaron entre septiembre y diciembre de 1984 dos campañas con el buque oceanográfico "El Austral" en el golfo San José y sur del golfo San Matías (provincia del Chubut, Argentina).

Conjuntamente con los datos de densidad de organismos, se contó con información sobre diversos factores ambientales, lo que permitió analizar las relaciones entre éstos y la distribución de las especies. Estos datos ambientales provinieron de las campañas antes citadas (profundidades, granulometría de sedimentos, presencia de fondos duros y de otros organismos), de otras campañas

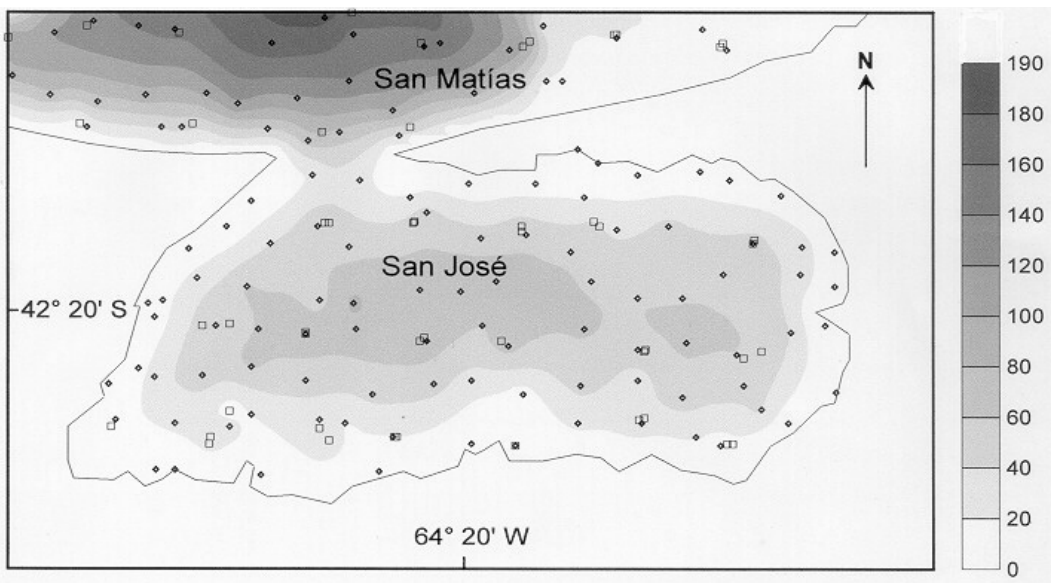

Figura 1

Golfo San José y sur del golfo San Matías. Ubicación de las estaciones de muestreo ( $\diamond$ : San Jose I y $\square$ : San José II).

San José Gulf and South of San Matías Gulf. Sampling stations $(\diamond$ : San José I and $\square$ : San José II).

previamente (Zaixso 1996). En cada una de las estaciones de muestreo se tomaron una o dos unidades muestrales (u.m.) con una draga tipo van Veen modificada, con una superficie de muestreo de $0,25 \mathrm{~m}^{2}$ y $100 \mathrm{Kg}$ de peso. En la primera de las campañas se muestreo un total de 122 estaciones a razón de una u.m. de draga por estación; en la segunda campaña se muestreo un total de 30 estaciones a razón de dos u.m. de draga por estación. En los sitios a los que la embarcación no tenía acceso, debido a la escasa profundidad, el muestreo se llevó a cabo mediante buceo autónomo, raspando el fondo o excavando con una pala y manteniendo el mismo tamaño de muestra.

Los equinodermos presentes fueron separados a bordo lavando las muestras con agua de mar sobre un tamiz de 1,5 mm de abertura y conservados en alcohol glicerinado. Para los efectos de los análisis que se indican más adelante, se seleccionaron las especies más frecuentes en el muestreo, presentes al menos en un 5\% de las unidades muestrales.

La densidad (individuos $\mathrm{m}^{-2}$ ) fue volcada sobre una carta del golfo a partir de un grillado obtenido por el método "point kriging" (Isaaks \& Srivastava 1989) con ayuda del programa SSTAT (Ingram 1995). Como matriz de datos se utilizaron la latitud, longitud y densidad de las diferentes especies en las estaciones de muestreo, incluyendo una serie adicional de valores de abundancia iguales a cero, correspondiente a puntos de la línea de costa del golfo, ya que ninguna de las especies estudiadas se encuentra normalmente por encima del nivel de la bajamar media.

Conjuntamente a las u.m. de equinodermos, se

El diseño general del muestreo fue expuesto 
dispuso de la siguiente información: profundidad, granulometría del sedimento, presencia de sustratos duros (toba) y de macroalgas, temperaturas de fondo para agosto y diciembre de 1984 y velocidad de las corrientes de superficie.

Las u.m. de sedimento fueron obtenidas con un cilindro de plexiglás de los $10 \mathrm{~cm}$ superficiales de las u.m. extraídas con la draga. En los muestreos efectuados con buzo, las u.m. de sedimento se tomaron directamente con el cilindro de plexiglás. En laboratorio, las u.m. de sedimento fueron secadas en estufa a $100{ }^{\circ} \mathrm{C}$ hasta peso constante, pesadas y luego separadas en húmedo en 7 fracciones correspondientes a las siguientes aberturas en milímetros (entre paréntesis se señalan los valores correspondientes en la escala Phi): 4,00 (-2); 2,00 (-1); $1,00(0) ; 0,50$ (1); 0,25 (2); 0,125 (3); 0,0625 (4). Las fracciones obtenidas fueron nuevamente secadas hasta peso constante y pesadas; la fracción de sedimentos finos (menores de $0,0625 \mathrm{~mm}$ de diámetro) fue estimada por diferencia respecto del peso seco inicial. Los valores obtenidos fueron expresados como porcentajes del total de la muestra.

Los fondos duros, consistentes principalmente en tobas calcáreas, fueron considerados sólo por su presencia. Este tipo de fondo se encontró casi con exclusividad en las u.m. obtenidas por buceo en aguas someras y la eficiencia del muestreo sobre los mismos se considera equiparable a la obtenida con draga sobre fondos blandos.

Las macroalgas, posible fuente de alimento para algunas de las especies estudiadas, fueron evaluadas a través de su presencia en las u.m. de los dragados.

Las temperaturas de fondo para agosto y diciembre de 1984, fueron estimadas de los datos obtenidos durante las campañas del Servicio Centralizado de Química del Centro Nacional Patagónico, llevadas a cabo previamente. Las temperaturas de fondo del mes de agosto se consideran representativas del patrón de temperaturas en invierno, en tanto que las temperaturas de fondo del mes de diciembre se consideran representativas del patrón de temperaturas de verano.

La intensidad de las corrientes de superficie en el golfo San José fue obtenida digitalizando las figuras del trabajo de Palma \& Serman (1984); los valores obtenidos fueron agrupados en las siguientes clases arbitrarias: $\mathrm{C} 1$ : de 0 a $0,33 \mathrm{~m} / \mathrm{s} ; \mathrm{C} 2$ : de 0,34 a $0,66 \mathrm{~m} / \mathrm{s}$; C3: más de $0,66 \mathrm{~m} / \mathrm{s}$.

El detalle de la distribución de los factores ambientales ha sido ilustrado en contribuciones anteriores (Zaixso 1996a, Zaixso et al. 1998), excepto las temperaturas las cuales son mostradas en las Figs. 2 y 3. Las profundidades a las que fueron obtenidas las u.m. son indicadas en la Fig. 1.
Con los dos conjuntos de datos (abundancias y factores ambientales) se llevó a cabo un análisis canónico de correspondencias (CCA), donde las u.m. con presencia de alguna de las especies de equinodermos seleccionadas (88 u.m.) fueron sometidas a una ordenación restringida en función de un conjunto limitado de variables ambientales. Las abundancias fueron transformadas a través de una transformación "piece-wise", donde los niveles de corte fueron 0.1, 2.0, $5.0,10.0,20.0$ y 100.0 (1 a 6). Las variables ambientales fueron: la profundidad (variable cuantitativa), el porcentaje de cada una de las fracciones granulométricas (variables cuantitativas), la presencia de sustratos duros (toba) como variable "dummy", la velocidad de corrientes (considerada como variable cuantitativa siguiendo la sugerencia de ter Braak (1995)), las temperaturas de fondo en agosto y diciembre de 1984 y finalmente la presencia de macroalgas que fue incluida también como variable de tipo nominal.

El análisis se llevó a cabo mediante el programa CANOCO en su versión 3.10 (ter Braak 1987 y 1990). Las variables ambientales fueron seleccionadas en razón de su importancia en la determinación de los datos de las especies con una selección tipo "forward", tomada cuenta de la ausencia de colinearidad entre las mismas (factores de inflación de la varianza menores de 10); se incluyeron sólo aquellas variables cuyo valor de $\mathrm{p}$ (estimado con una simulación de Monte Carlo con 999 permutaciones) fuera menor o igual que $\alpha=0,15$. La conveniencia de usar un modelo unimodal (CCA) en lugar de un modelo monotónico (análisis de redundancias) fue sugerida por los resultados obtenidos en un análisis preliminar (correspondencias sin tendencias) donde se obtuvo que el largo (gradiente) de los ejes de ordenación I y II fue mayor que 4 desvíos estándar (ter Braak 1995: 154).

En los diagramas de ordenación del CCA la proyección de los puntos de las especies sobre las flechas de variables ambientales representan aproximadamente la ubicación del rango de los promedios ponderados de la especie sobre esa variable (ter Braak 1995). Otras reglas de interpretación de los diagramas obtenidos en el CCA son consideradas con detalle en ter Braak (1995).

Dado que los promedios ponderados corresponden a óptimos de la especie, sólo en el caso de que la curva respuesta de la abundancia de ésta corresponda a un modelo unimodal simétrico (respuesta gaussiana no sesgada), se llevó a cabo y como complemento del CCA, un ajuste a modelos jerárquicos Huisman-OlffFresco (HOF) de las curvas respuesta de las abundancias (transformadas) en función de cada uno de los factores ambientales seleccionados por el CCA 


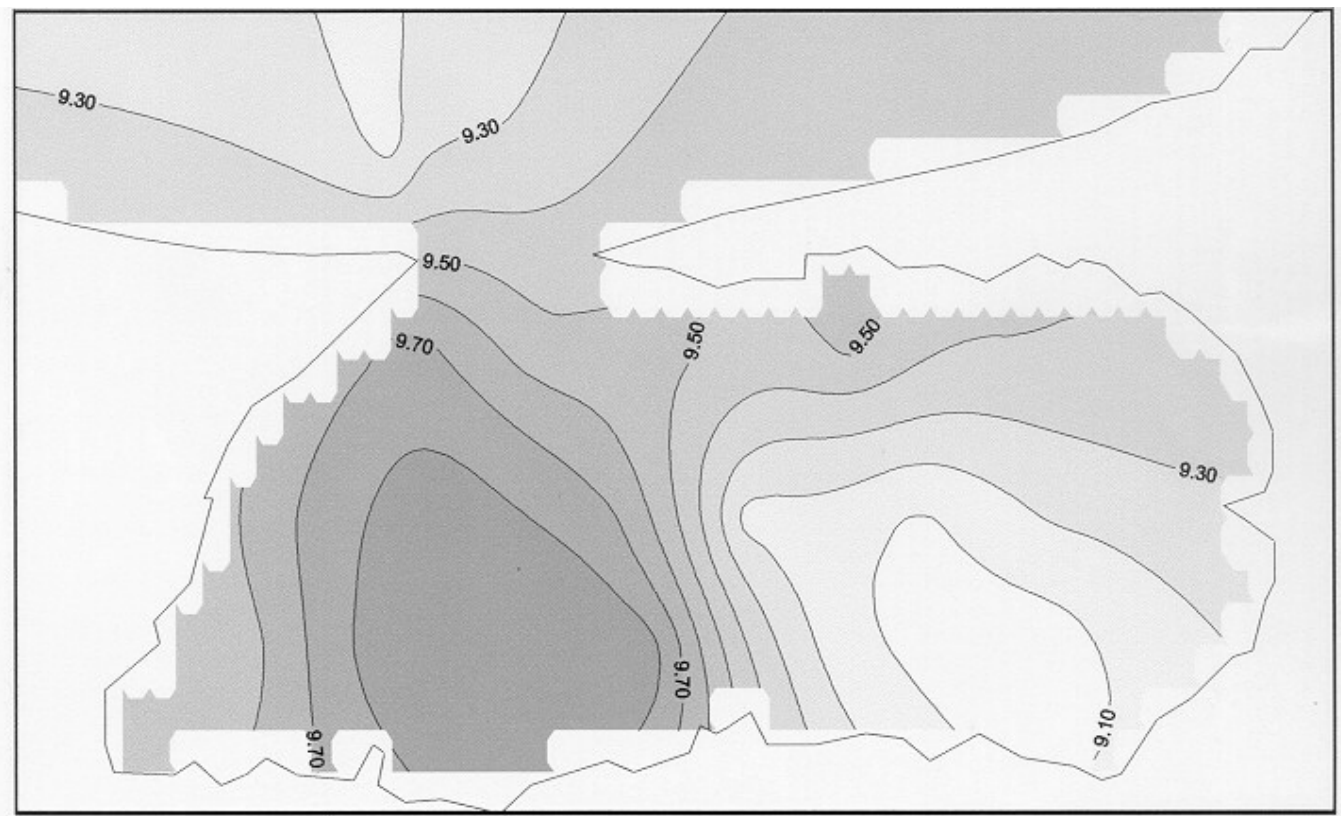

Figura 2

Temperaturas $\left({ }^{\circ} \mathrm{C}\right)$ de fondo en agosto de 1984.

August - 1984 bottom temperatures.

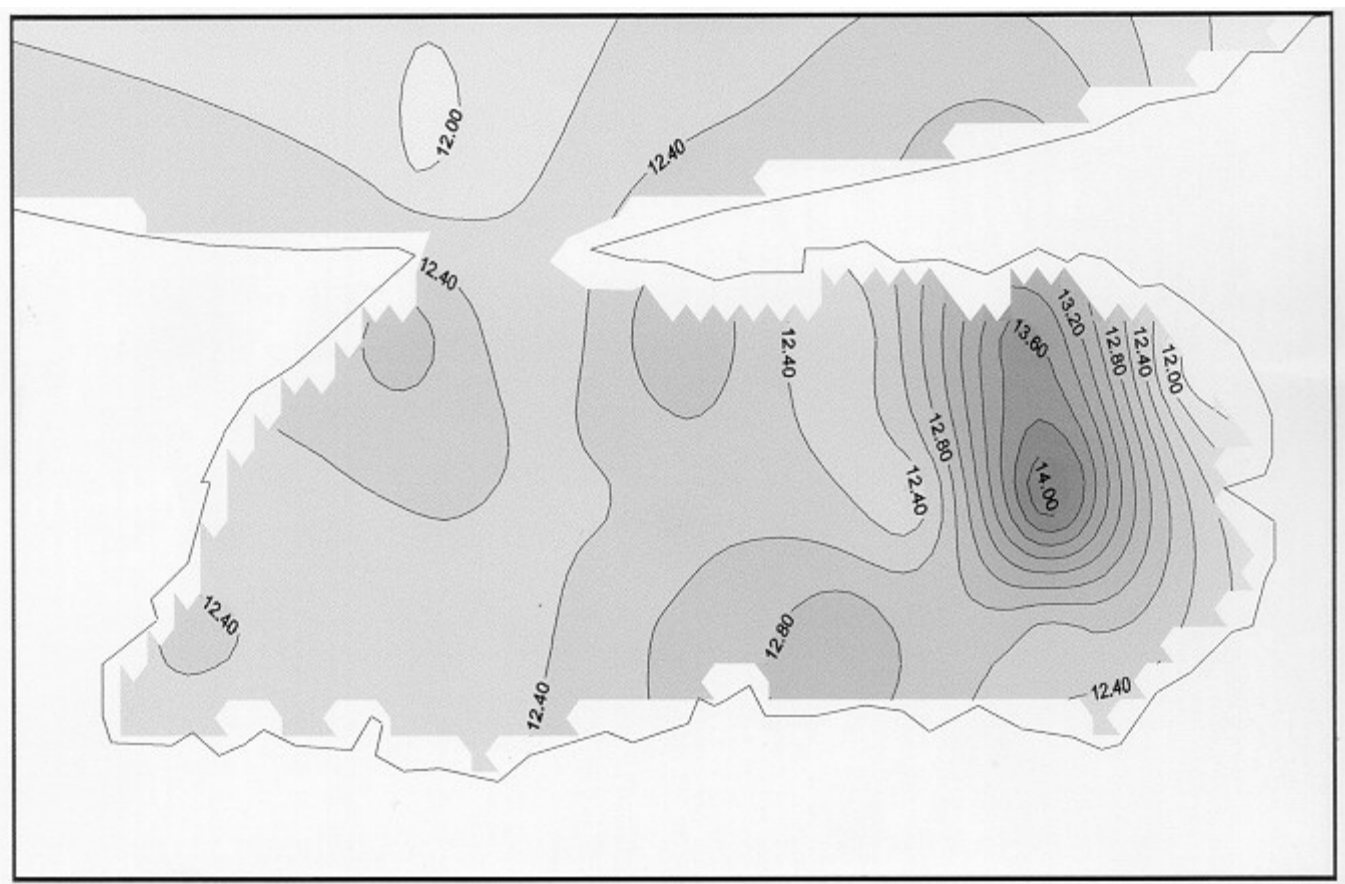

Figura 3

Temperaturas $\left({ }^{\circ} \mathrm{C}\right)$ de fondo en diciembre de 1984.

December - 1984 bottom temperatures. 

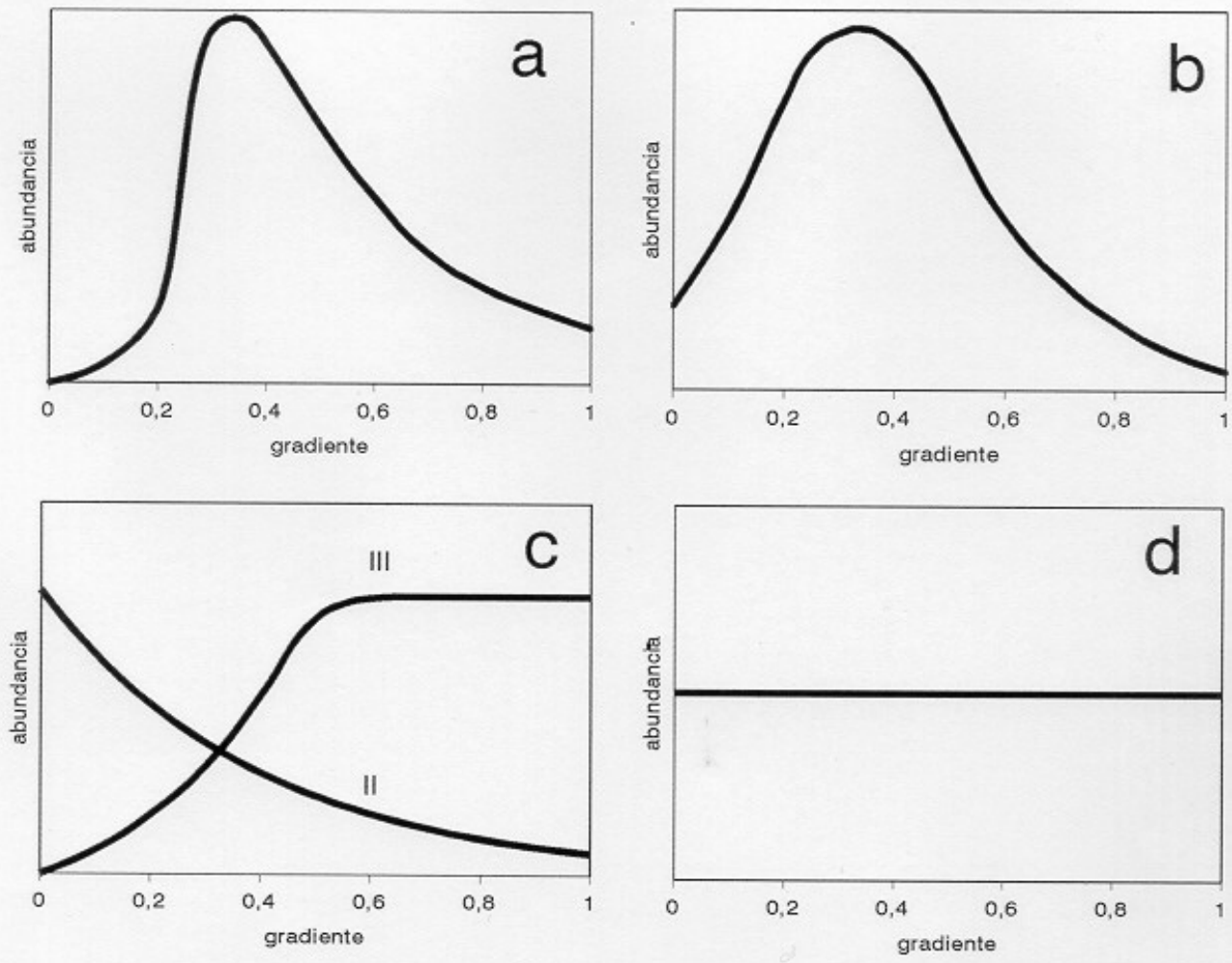

Figura 4

Modelos jerárquicos HOF. a, modelo V (respuesta unimodal asimétrica); b, modelo IV (respuesta unimodal simétrica); c, modelo II (respuesta monótona) y modelo III (respuesta monótona con asíntota); d, modelo I (respuesta uniforme).

HOF hierarchical models: a, . a, model V (skewed unimodal response); b, model IV (symmetric unimodal response); c, model II (monotone response) y model III (monotone response with a plateau); d, model I (flat response).

(Huisman et al. 1993). Los modelos HOF permiten discriminar entre las posibles curvas respuesta de una especie respecto de una variable ambiental particular de acuerdo a cinco modelos jerárquicos que son probados secuencialmente por su significación. Por modelos jerárquicos se entiende que el modelo más simple posee (1) menor número de parámetros que los más complejos, y (2) puede ser derivado de los modelos más complejos fijando algunos parámetros (Huisman et al. 1993). Los modelos propuestos son (Fig. 4): Modelo I (respuesta uniforme), Modelo II (respuesta monótona), Modelo III (respuesta monótona con asíntota), Modelo IV (respuesta unimodal simétrica) y Modelo V (respuesta unimodal asimétrica). El modelo más complejo es la respuesta unimodal asimétrica, cuya función es:

$$
\mu(\mathrm{a}, \mathrm{b}, \mathrm{c}, \mathrm{d}, \mathrm{x}, \mathrm{M})=\mathrm{M}(1 /(1+\exp (\mathrm{a}+\mathrm{bx})))(1 /(1+\exp (\mathrm{c}-\mathrm{dx})))
$$

Donde $\mu$ es el valor esperado (de abundancia) y es dependiente de: i. Los valores máximo y mínimo muestreados de la variable ambiental gradiente

$\mathrm{X}_{\text {proporcional al rango del gradiente }}=\mathrm{X}-\mathrm{X}_{\text {mín. }} / \mathrm{X}_{\text {máx. }}-\mathrm{X}_{\text {mín. }}$

ii. Del valor máximo posible (de abundancia) en el muestreo.

iii. De cuatro parámetros a estimar $(\mathrm{a}, \mathrm{b}, \mathrm{c}, \mathrm{d})$. El modelo $\mathrm{V}$ se simplifica en el modelo IV fijando $\mathrm{d}$ $=\mathrm{b}$; en el modelo III fijando $\mathrm{d}=0$; en el modelo II fijando $\mathrm{c}=-\infty \quad \mathrm{y} \quad \mathrm{d}=0$ y en el modelo I fijando $\mathrm{b}$ $=0, \mathrm{c}=-\infty \mathrm{y} \mathrm{d}=0$.

El ajuste fue llevado cabo con el programa HOF versión 2.3 (Oksanen 1996 y 1998), trabajando con un nivel de significación $\alpha=0,05$, error Poisson y $\mathrm{M}=6$.

El programa lleva a cabo el ajuste utilizando una estimación de máxima verosimilitud (con error Poisson). Los parámetros son no lineales y se estiman por iteración utilizando una variante de los métodos 
quasi-Newton (Oksanen 1996). Los modelos son probados secuencialmente, siendo el método equivalente al usado en regresión múltiple con "modelos completos" y "modelos reducidos" alternativos: si se tienen dos modelos alternativos donde uno tiene un parámetro más que el otro y la diferencia de ajuste (estimada con un estadístico apropiado) entre ambos excede un determinado valor crítico (a un nivel de significación $\alpha$ ), entonces el "parámetro accesorio" es significativo y debe adoptarse el modelo que no puede ser simplificado sin un cambio significativo en el valor del estadístico que mide las diferencias de ajuste (Oksanen 1996). La versión 2.3 del programa utiliza una prueba de $\mathrm{F}$ para el análisis de la significación de las diferencias de desvíos, la que es más apropiada para datos de tipo sobredisperso que la prueba de $\chi^{2}$ utilizada en versiones previas (Anderson 1988). El estadístico $\mathrm{F}$ se calcula (Oksanen 1998) a partir de los desvíos como:

$\mathrm{F}_{1, \mathrm{n}-4}=\left(\left(\mathrm{D}_{\mathrm{i}}-\mathrm{D}_{\mathrm{j}}\right) / \mathrm{D}_{\mathrm{v}}\right)(\mathrm{n}-4)$

$\mathrm{D}_{\mathrm{i}}$ : desvío del modelo con $\mathrm{p}$ parámetros

$\mathrm{D}_{\mathrm{j}}$ : desvío del modelo con $\mathrm{p}-1$ parámetros

$\mathrm{D}_{\mathrm{v}}$ : desvío del modelo "completo" $\mathrm{V}$ (sea cual fuere el modelo a probar)

En primer lugar se ajustan y prueban los modelos $\mathrm{V}$ y IV; si el modelo IV no es aceptado, entonces se ajusta el modelo III y se lo prueba contra el modelo V; en consecuencia el modelo $\mathrm{V}$ es aceptado sólo si es mejor que los modelos IV y III. Si es aceptado el modelo IV o bien el modelo III, entonces éste es probado contra el modelo II. Finalmente de ser aceptado el modelo II el mismo es probado contra el modelo I. En la salida del programa se grafican automáticamente los modelos que presentan el mejor ajuste para el nivel de significación elegido.

\section{Resultados}

\section{a. Distribución general en los golfos}

Las especies más frecuentes (presentes al menos en 5\% de las u.m.) en el área de estudio son: los equinoideos Trypilaster philippii (Gray 1851), Pseudechinus magellanicus (Philippi 1857) y Arbacia dufresnei (Blainville 1825); los ofiuroideos Ophioplocus januarii (Lütken 1856), Ophiactis asperula (Philippi 1858), Amphiura crassipes Ljungman 1867 y Amphiura magellanica Ljungman 1867 y los holoturoideos Chiridota marenzelleri Perrier 1904 y Pentamera chiloensis (Ludwig 1886).

De acuerdo a las gráficas de distribución obtenidas por el método "point kriging" y volcadas en las Figs. 5 a 13 se puede observar que, en general, las especies de equinodermos estudiadas se hallan ausentes en la mitad oriental del golfo San José.



Figura 5

Pseudechinus magellanicus (Philippi): densidad de individuos por $\mathbf{m}^{2}$.

Pseudechinus magellanicus (Philippi): Number of individuals $/ \mathrm{m}^{2}$. 


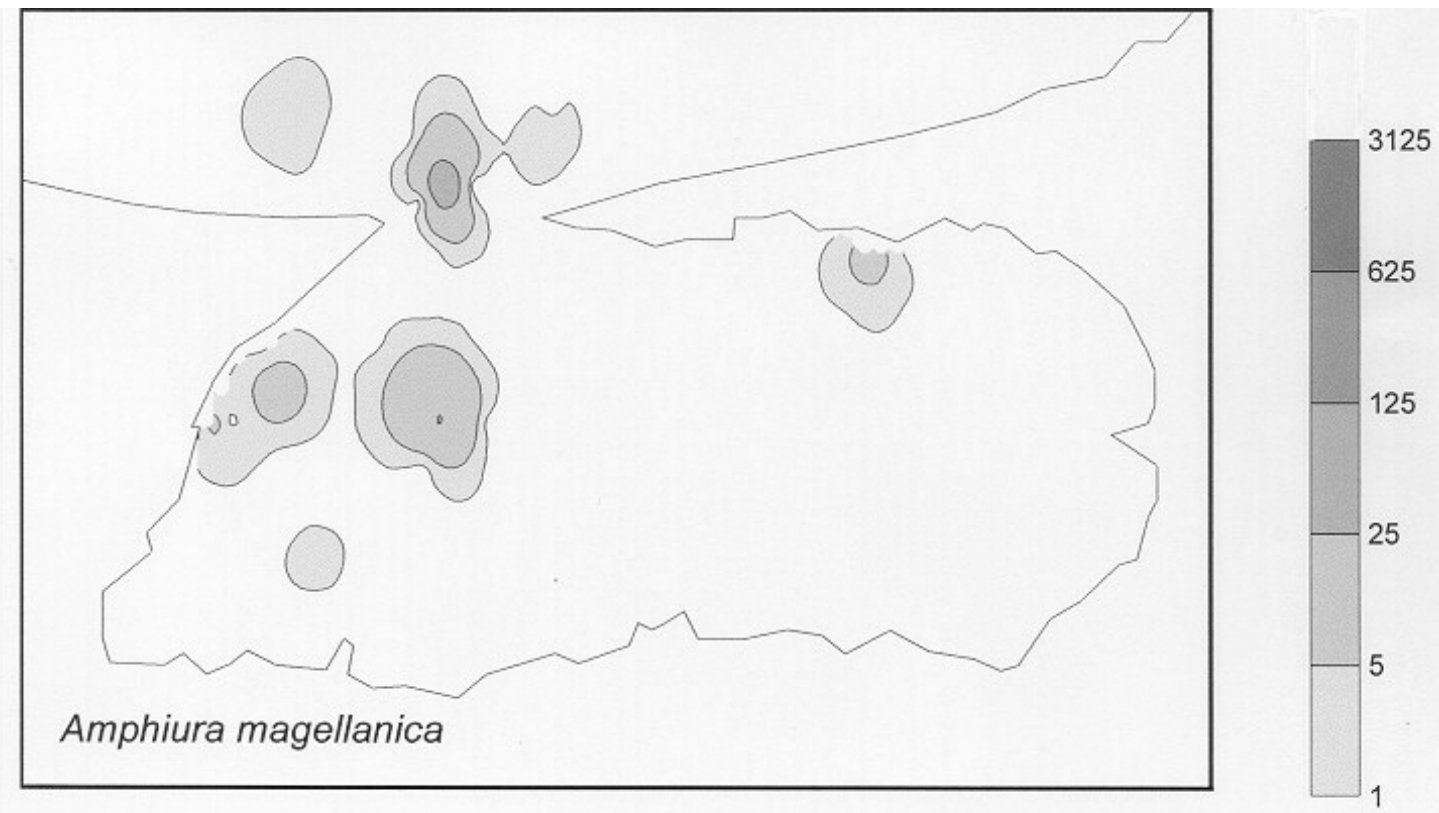

Figura 6

Amphiura magellanica Ljungman: densidad de individuos por $\mathbf{m}^{2}$. Amphiura magellanica Ljungman: Number of individuals / $\mathrm{m}^{2}$.
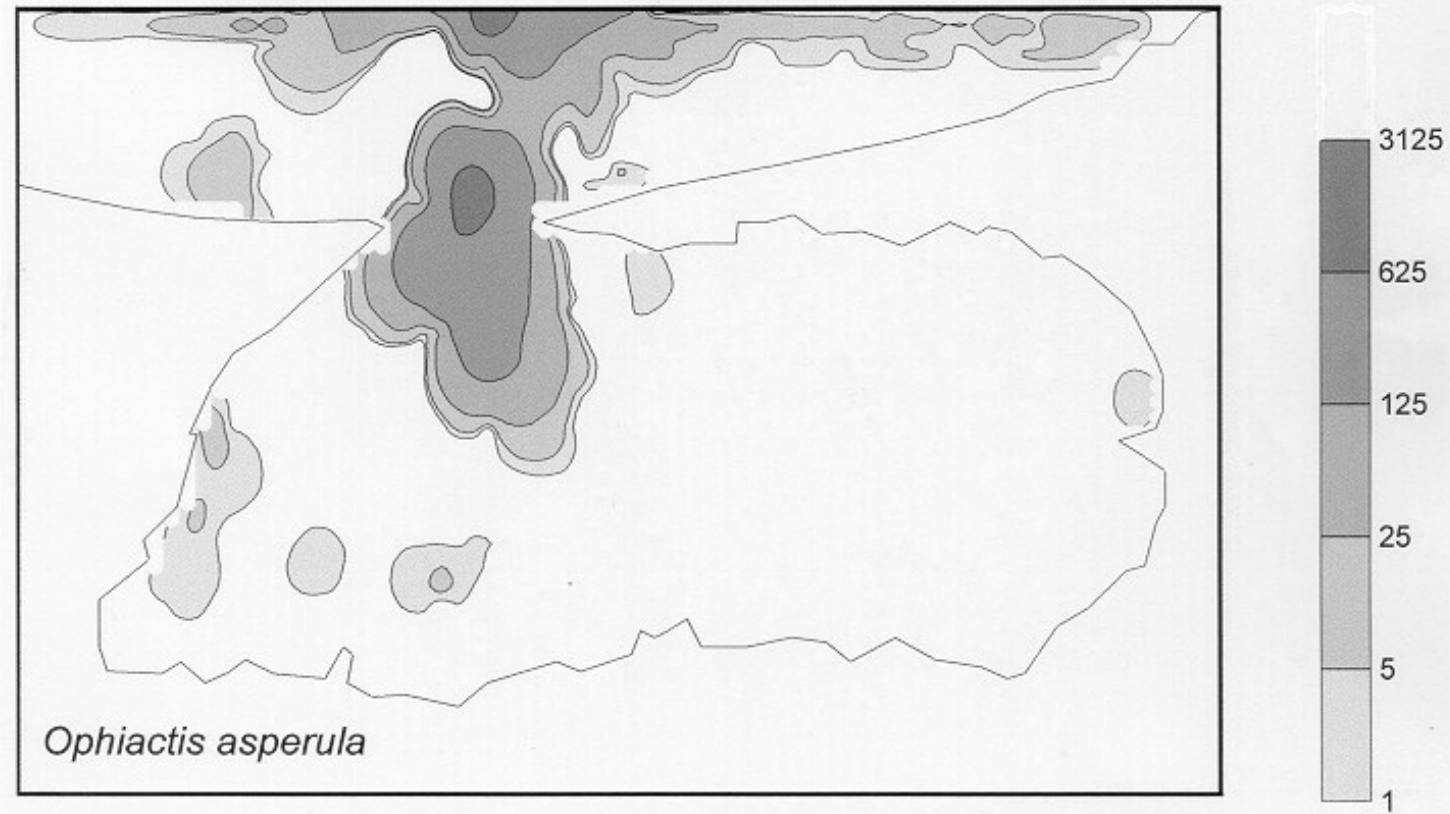

Figura 7

Ophiactis asperula (Philippi): densidad de individuos por $\mathbf{m}^{2}$.

Ophiactis asperula (Philippi): Number of individuals $/ \mathrm{m}^{2}$. 




Figura 8

Arbacia dufresnei (Blainville): densidad de individuos por $\mathbf{m}^{2}$. Arbacia dufresnei (Blainville): Number of individuals $/ \mathrm{m}^{2}$.
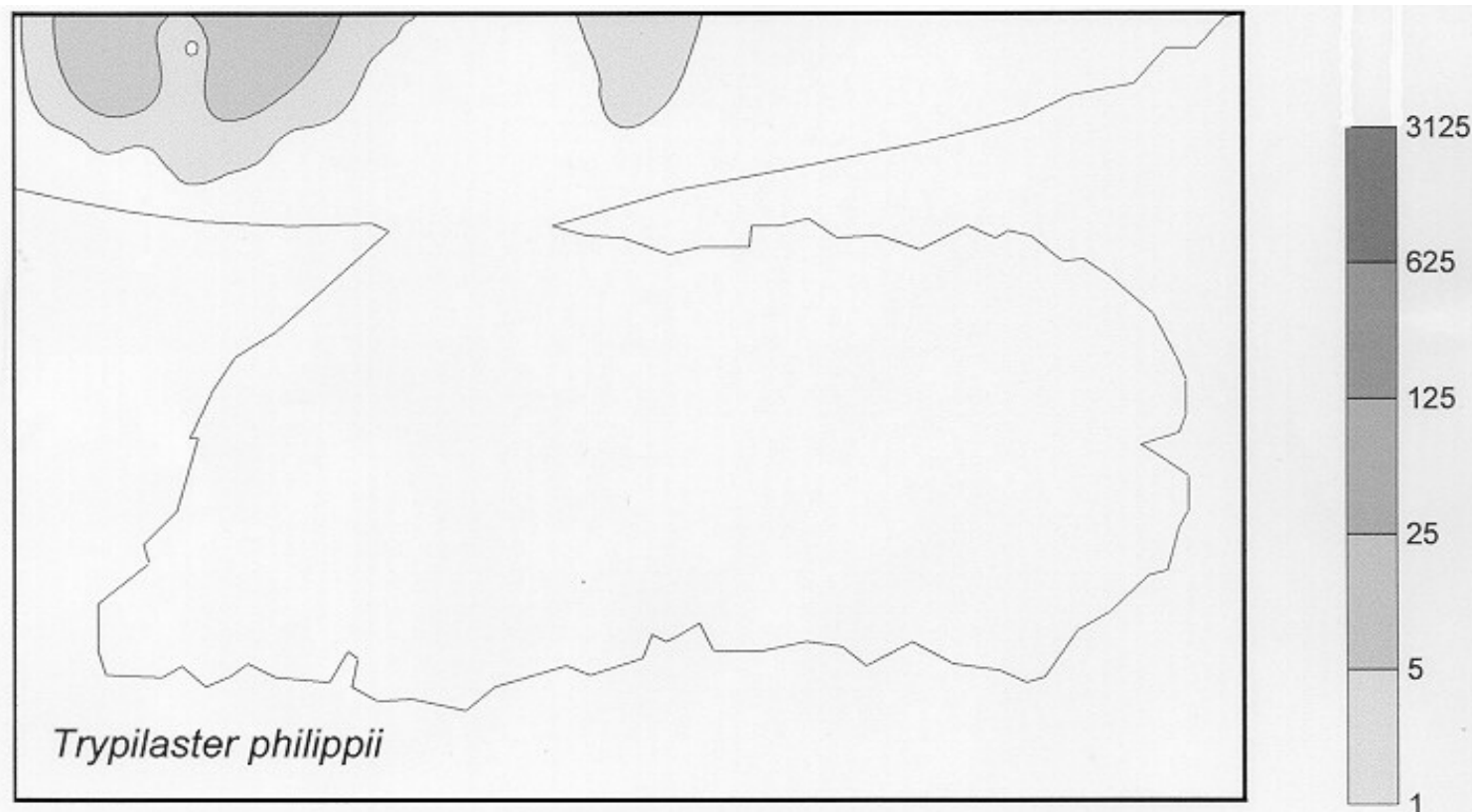

Figura 9

Trypilaster philippii (Gray): densidad de individuos por $\mathbf{m}^{2}$.

Trypilaster philippii (Gray): Number of individuals / $\mathrm{m}^{2}$. 

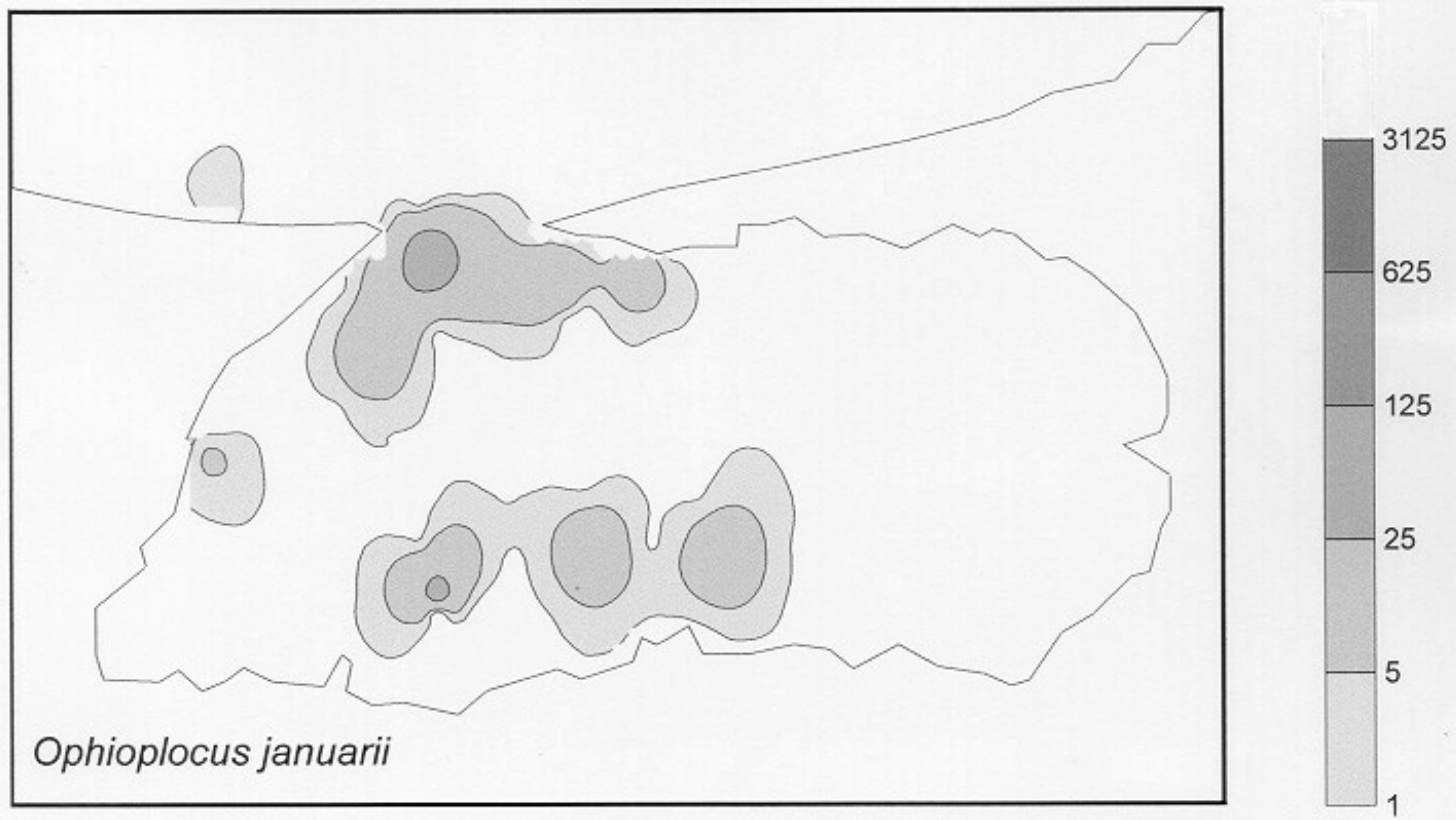

Figura 10

Ophioplocus januarii (Lütken): densidad de individuos por $\mathbf{m}^{\mathbf{2}}$.

Ophioplocus januarii (Lütken): Number of individuals / $\mathrm{m}^{2}$.

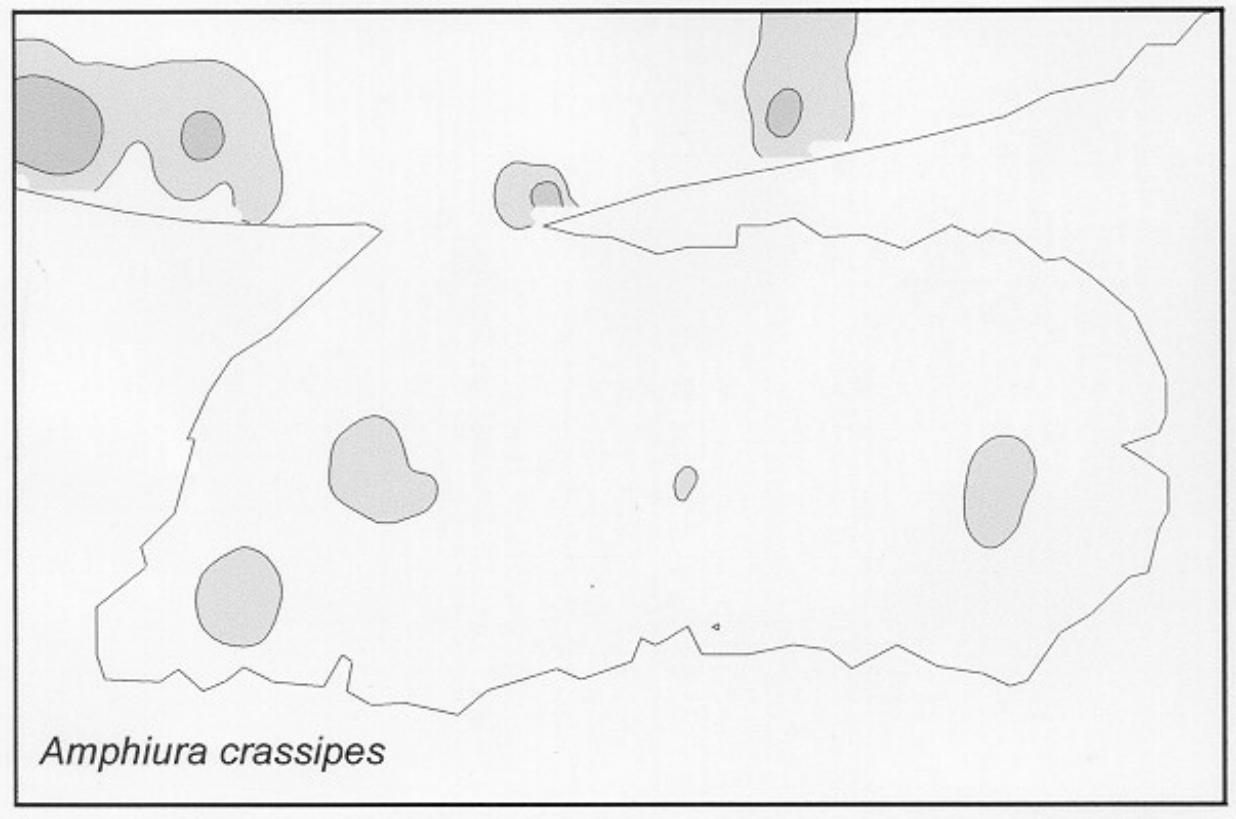

3125

625

Figura 11

Amphiura crassipes Ljungman: densidad de individuos por $\mathbf{m}^{2}$.

Amphiura crassipes Ljungman: Number of individuals / $\mathrm{m}^{2}$. 

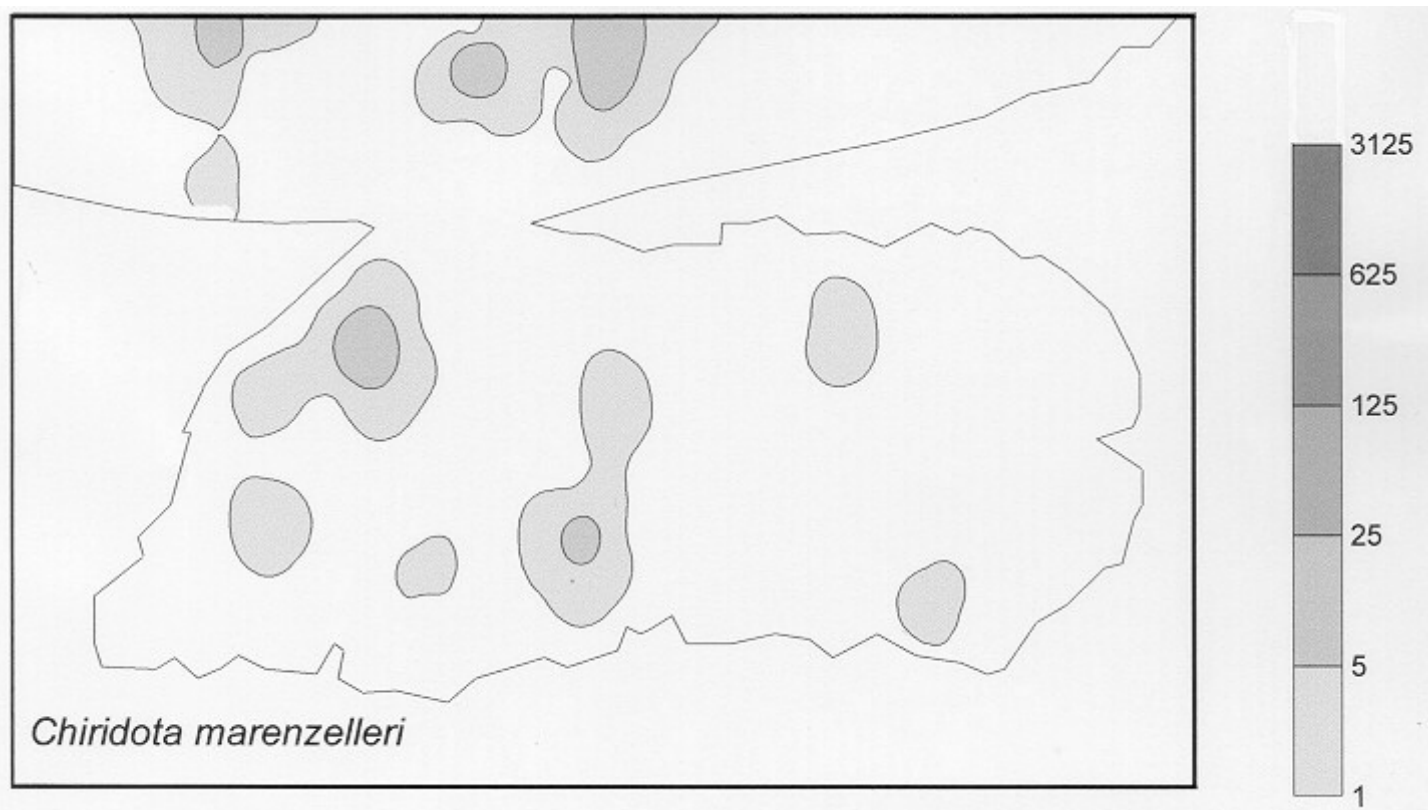

Figura 12

Chiridota marenzelleri Perrier: densidad de individuos por $\mathbf{m}^{2}$.

Chiridota marenzelleri Perrier: Number of individuals $/ \mathrm{m}^{2}$.

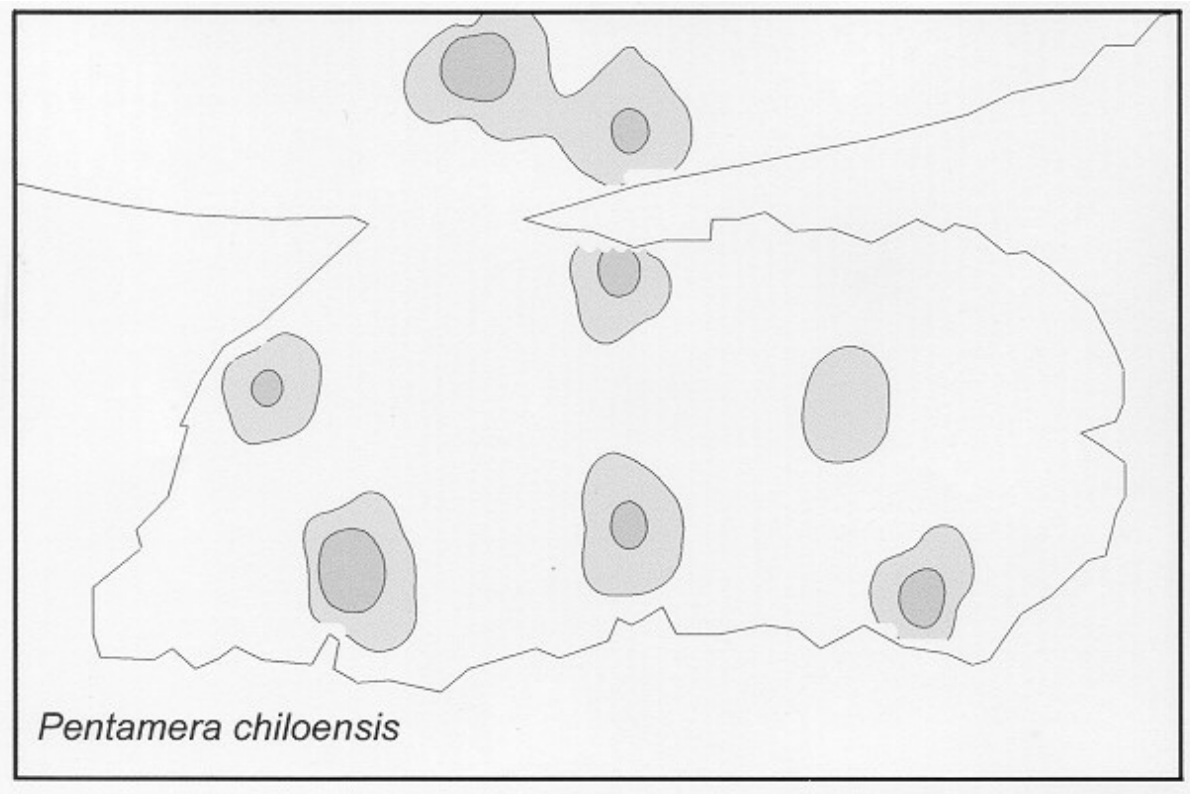

Figura 13

Pentamera chiloensis (Ludwig): densidad de individuos por $\mathbf{m}^{2}$.

Pentamera chiloensis (Ludwig): Number of individuals $/ \mathrm{m}^{2}$. 
Las especies Pseudechinus magellanicus (de 2 a 185 $\mathrm{m}$ de profundidad), Amphiura magellanica (2 a $105 \mathrm{~m}$ ) y Ophiactis asperula (2 a $185 \mathrm{~m})$, se hallan particularmente asociadas a una zona aproximadamente perpendicular a la línea de la boca del golfo San José y que coincide con la distribución de las u.m. con dominancia de sedimentos gruesos y las corrientes mayores de 0,66 m/s (Fig. 5 a 7). Pseudechinus magellanicus y Ophiactis asperula son las especies más abundantes en la zona de estudio; la última de las nombradas alcanza densidades de más de 1000 individuos por $\mathrm{m}^{2}$ en el área de la desembocadura del golfo San José.

El erizo Arbacia dufresnei se ubica preferentemente en la zona costera del golfo, desde el nivel de bajamares hasta los $68 \mathrm{~m}$ de profundidad; sus máximas densidades se hallan sobre la costa oeste y en la zona de la desembocadura (Fig. 8).

El erizo Trypilaster philippii se halla limitado a la zona profunda del golfo San Matías, entre los 95 y 158 $m$ de profundidad (Fig. 9).
El ofiuroideo Ophioplocus januarii presenta sus máximas abundancias en la zona costera sur del golfo San José y en la zona de la desembocadura, se lo encuentra a profundidades entre 5 y $51 \mathrm{~m}$ (Fig. 10).

Las especies Amphiura crassipes (de 3 a 120 m de profundidad), Chiridota marenzelleri (22 a $158 \mathrm{~m})$ y Pentamera chiloensis (5 a $154 \mathrm{~m}$ ), presentan un patrón de distribución poco claro y se encuentran dispersas por toda el área muestreada (Figs. 11 a 13).

\section{b. Relaciones generales en el análisis canónico de correspondencias (CCA)}

Los resultados del CCA (Fig. 14) se muestran en un diagrama de ordenación de especies y variables ambientales seleccionadas por el análisis, las cuales fueron (valores de $\mathrm{p}$ estimados entre paréntesis): limosarcillas $(p=0,001)$, arenas muy finas $(p=0,001)$, profundidad $\quad(p=0,001), \quad$ corrientes $\quad(p=0,001)$, temperaturas de "verano" $(\mathrm{p}=0,038)$ y arenas muy gruesas $(\mathrm{p}=0,146)$.

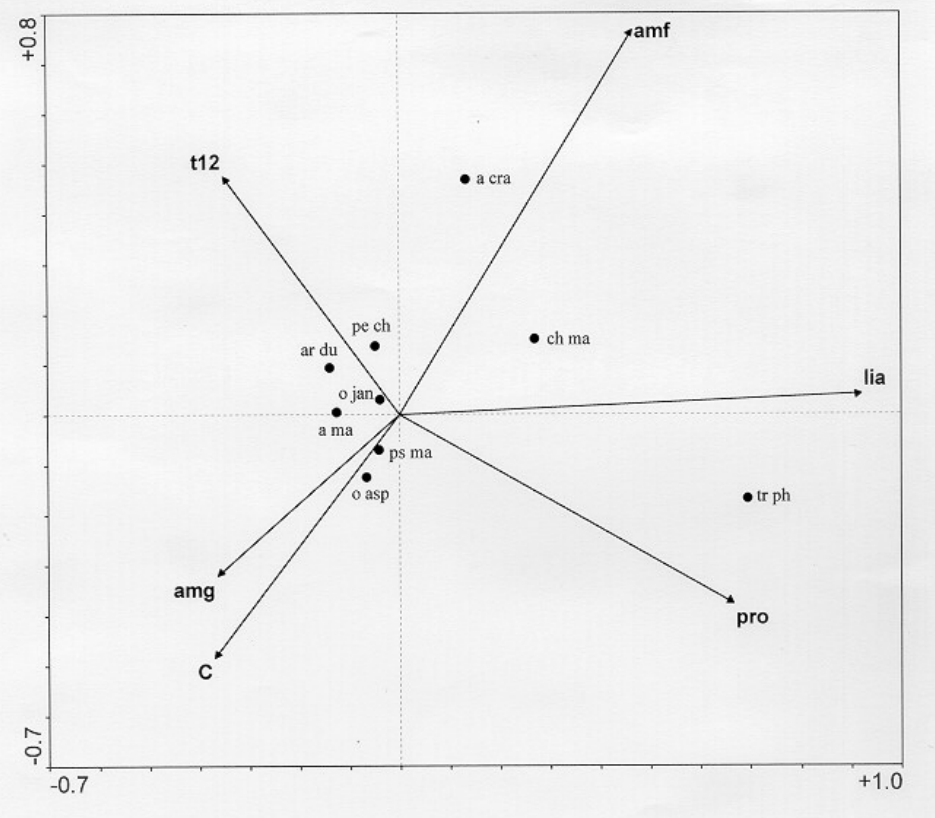

Figura 14

Diagrama de ordenación del CCA: especies (puntos) y variables ambientales (flechas). a cra, Amphiura crassipes; ar du, Arbacia dufresnei; a ma, Amphiura magellanica; ch ma, Chiridota marenzelleri; o asp, Ophiactis asperula; o jan, Ophioplocus januarii; pe ch, Pentamera chiloensis; ps ma, Pseudechinus magellanicus; tr ph, Trypilaster philippii; amf, arenas muy finas; amg, arenas muy gruesas; C, corrientes; lia, limos-arcillas; pro, profundidad; t12, temperatura de fondo en diciembre.

CCA ordination diagram: Species (points) and environmental variables (arrows). a cra, Amphiura crassipes; ar du, Arbacia dufresnei; a ma, Amphiura magellanica; ch ma, Chiridota marenzelleri; o asp, Ophiactis asperula; o jan, Ophioplocus januarii; pe ch, Pentamera chiloensis; ps ma, Pseudechinus magellanicus; tr ph, Trypilaster philippii; amf, very fine sands; amg, very coarse sands; C, currents; lia, silt-clay; pro, depth; t12, December bottom temperature. 
El eje I se halla correlacionado en forma positiva con el porcentaje de limos-arcillas $(\mathrm{r}=0,921)$, con la profundidad $(\mathrm{r}=0,664)$ y el porcentaje de arenas muy finas $(r=0,467)$ y negativamente con el porcentaje de arenas muy gruesas $(\mathrm{r}=-0,363)$, las corrientes de superficie $(\mathrm{r}=-0,371)$ y las temperaturas de "verano" $(\mathrm{r}=-0,349)$. El eje II se correlaciona positivamente con las arenas muy finas $(\mathrm{r}=0,764)$ y las temperaturas de verano $(\mathrm{r}=0,474)$ y negativamente con las corrientes de superficie $(\mathrm{r}=-0,483)$, la profundidad $(\mathrm{r}=-0,377)$ y las arenas muy gruesas $(\mathrm{r}=-0,320)$. La fracción de limosarcillas se halla correlacionada positivamente con la fracción de arenas muy finas $(r=0,427)$ y con la profundidad $(\mathrm{r}=0,404)$ y negativamente con las arenas muy gruesas $(r=-0,422)$ y las corrientes $(r=-0,409)$. La profundidad se halla correlacionada negativamente con las temperaturas de verano $(\mathrm{r}=-0,602)$.

De acuerdo con lo anterior, las especies con "scores" positivos altos con el eje I como Trypilaster philippii se hallan restringidas a sustratos con dominancia de limosarcillas, profundidades altas y corrientes de superficie débiles. Amphiura crassipes con un "score" alto y positivo sobre el eje II se relaciona con fondos con dominancia de arenas muy finas y con temperaturas de fondo altas en verano. Especies como Ophioplocus januarii, ubicadas hacia el origen de los ejes no son afectadas por los factores citados o bien están restringidas a valores intermedios de los mismos.

Los resultados obtenidos con el CCA se hallan resumidos en la Tabla 1.

\section{c. CCA y modelos HOF}

A continuación se describe el diagrama de ordenación de especies y variables ambientales en relación a la distribución de sus promedios ponderados (ubicación del rango de las proyecciones perpendiculares de los puntos de especies) sobre las flechas de las variables ambientales y haciendo referencia al modelo HOF más ajustado (Fig. 4) para cada variable ambiental seleccionada por el CCA. Los resultados del ajuste a modelos HOF son resumidos en la Tabla 2.

Tabla 1

Resultados del CCA.

a. Promedio ponderado, desviación estándar (s) y factor de inflación de la varianza (VIF) de las variables ambientales seleccionadas en el CCA: amg, arenas muy gruesas (\%); amf, arenas muy finas (\%); lia, limosarcillas (\%); pro, profundidad (m); C, corrientes (rango 1-3); $t_{12}$, temperatura de fondo en diciembre $\left({ }^{\circ} \mathrm{C}\right)$.

CCA Results.

a. Weighted mean, standard deviation (s) and variance inflation factor (VIF) of CCA selected environmental variables: amg, very coarse sands (\%); amf, very fine sands $(\%)$; lia, silt-clay $(\%)$; pro, depth $(\mathrm{m})$; $\mathrm{C}$, currents (range 1-3); $\mathrm{t}_{12}$, December bottom temperature $\left({ }^{\circ} \mathrm{C}\right)$.

\begin{tabular}{lccc}
\hline Nombre & Promedio ponderado & s & VIF \\
& & & \\
amg & 8,9844 & 10,3696 & 1,6054 \\
amf & 13,8591 & 16,5500 & 1,4796 \\
lia & 19,9879 & 25,0186 & 2,3983 \\
pro & 57,7074 & 48,0306 & 2,7625 \\
C & 2,0429 & 0,9140 & 1,8494 \\
$\mathrm{t}_{12}$ & 12,3556 & 0,1763 & 1,6187 \\
\hline
\end{tabular}

b. Sumario del CCA.

b. CCA summary.

\begin{tabular}{|c|c|c|c|c|c|c|}
\hline Ejes & & 1 & 2 & 3 & 4 & Inercia total \\
\hline Valores propios & & 0,445 & 0,281 & 0,206 & 0,108 & 3,882 \\
\hline Correlaciones especies-ambiente & & 0,790 & 0,655 & 0,669 & 0,532 & \\
\hline \multirow[t]{2}{*}{ Porcentaje acumulado de la varianza } & de los datos de las especies & 11,5 & 18,7 & 24,0 & 26,8 & \\
\hline & de la relación especies -ambiente & 40,6 & 66,3 & 85,1 & 95,0 & \\
\hline $\begin{array}{l}\text { Suma de los valores propios no } \\
\text { restringidos }\end{array}$ & & & & & & 3,882 \\
\hline $\begin{array}{l}\text { Suma de los valores propios } \\
\text { canónicos }\end{array}$ & & & & & & 1,095 \\
\hline
\end{tabular}


Tabla 2

Ajuste a modelos jerárquicos $\mathrm{HOF}(\mathbf{p}<\mathbf{0 , 0 5})$ de las curvas respuesta de abundancia (transformación piece-wise) en función de las variables ambientales seleccionados por el CCA (forward): lia, limos-arcillas (\%); amf, arenas muy finas (\%); pro, profundidad (m); $C$, corrientes (rango 1-3); amg, arenas muy gruesas (\%); $t_{12}$, temperatura de fondo en diciembre $\left({ }^{\circ} \mathrm{C}\right)$. Se indican los estimadores de los modelos ajustados (ver texto).

Hierarchical HOF models fits $(\mathrm{p}<0.05)$ of species abundance response curves (piece-wise transformation) in function of CCA selected environmental variables (forward): lia, silt-clay (\%); amf, very fine sands (\%); pro, depth (m); C, currents (range 1-3); amg, very coarse sands $(\%)$; $\mathrm{t}_{12}$, December bottom temperature $\left({ }^{\circ} \mathrm{C}\right)$. The estimated parameters of adjusted models are indicated (see text).

\begin{tabular}{|c|c|c|c|c|c|c|}
\hline Especie & lia & amf & pro & $\mathbf{C}$ & amg & $t_{12}$ \\
\hline Pentamera chiloensis & $\begin{array}{l}\text { Modelo I } \\
a=3,47 \\
b=0 \\
c=-\infty \\
d=0\end{array}$ & $\begin{array}{l}\text { Modelo I } \\
a=3,47 \\
b=0 \\
c=-\infty \\
d=0\end{array}$ & $\begin{array}{l}\text { Modelo I } \\
a=3,47 \\
b=0 \\
c=-\infty \\
d=0\end{array}$ & $\begin{array}{l}\text { Modelo I } \\
\mathrm{a}=3,47 \\
\mathrm{~b}=0 \\
\mathrm{c}=-\infty \\
\mathrm{d}=0\end{array}$ & $\begin{array}{l}\text { Modelo I } \\
a=3,47 \\
b=0 \\
c=-\infty \\
d=0\end{array}$ & $\begin{array}{l}\text { Modelo I } \\
a=3,47 \\
b=0 \\
c=-\infty \\
d=0\end{array}$ \\
\hline $\begin{array}{l}\text { Chiridota } \\
\text { marenzelleri }\end{array}$ & $\begin{array}{l}\text { Modelo IV } \\
\mathrm{a}=-4,34 \\
\mathrm{~b}=8,19 \\
\mathrm{c}=5,17 \\
\mathrm{~d}=8,19\end{array}$ & $\begin{array}{l}\text { Modelo IV } \\
a=-5,13 \\
b=11,59 \\
c=6,07 \\
d=11,59\end{array}$ & $\begin{array}{l}\text { Modelo II } \\
a=4,09 \\
b=-1,98 \\
c=-\infty \\
d=0\end{array}$ & $\begin{array}{l}\text { Modelo I } \\
a=3,34 \\
b=0 \\
c=-\infty \\
d=0\end{array}$ & $\begin{array}{l}\text { Modelo I } \\
a=3,34 \\
b=0 \\
c=-\infty \\
d=0\end{array}$ & $\begin{array}{l}\text { Modelo I } \\
a=3,34 \\
b=0 \\
c=-\infty \\
d=0\end{array}$ \\
\hline Ophioplocus januarii & $\begin{array}{l}\text { Modelo I } \\
a=2,92 \\
b=0 \\
c=-\infty \\
d=0\end{array}$ & $\begin{array}{l}\text { Modelo II } \\
a=2,33 \\
b=2,78 \\
c=-\infty \\
d=0\end{array}$ & $\begin{array}{l}\text { Modelo IV } \\
a=-2,29 \\
b=18,53 \\
c=3,39 \\
d=18,53\end{array}$ & $\begin{array}{l}\text { Modelo I } \\
a=2,92 \\
b=0 \\
c=-\infty \\
d=0\end{array}$ & $\begin{array}{l}\text { Modelo IV } \\
a=-0,87 \\
b=7,95 \\
c=2,60 \\
d=7,95 \\
\end{array}$ & $\begin{array}{l}\text { Modelo IV } \\
a=-4,66 \\
b=9,59 \\
c=6,09 \\
d=9,59\end{array}$ \\
\hline Ophiactis asperula & $\begin{array}{l}\text { Modelo II } \\
a=1,08 \\
b=3,04 \\
c=-\infty \\
d=0 \\
\end{array}$ & $\begin{array}{l}\text { Modelo II } \\
a=0,90 \\
b=3,26 \\
c=-\infty \\
d=0\end{array}$ & $\begin{array}{l}\text { Modelo I } \\
a=1,61 \\
b=0 \\
c=-\infty \\
d=0\end{array}$ & $\begin{array}{l}\text { Modelo II } \\
a=3,38 \\
b=-3,21 \\
c=-\infty \\
d=0\end{array}$ & $\begin{array}{l}\text { Modelo I } \\
a=1,61 \\
b=0 \\
c=-\infty \\
d=0 \\
\end{array}$ & $\begin{array}{l}\text { Modelo II } \\
a=-0,02 \\
b=4,75 \\
c=-\infty \\
d=0\end{array}$ \\
\hline $\begin{array}{l}\text { Amphiura } \\
\text { magellanica }\end{array}$ & $\begin{array}{l}\text { Modelo II } \\
a=2,51 \\
b=5,36 \\
c=-\infty \\
d=0\end{array}$ & $\begin{array}{l}\text { Modelo II } \\
a=2,58 \\
b=3,20 \\
c=-\infty \\
d=0\end{array}$ & $\begin{array}{l}\text { Modelo I } \\
a=3,23 \\
b=0 \\
c=-\infty \\
d=0\end{array}$ & $\begin{array}{l}\text { Modelo II } \\
a=3,95 \\
b=-1,48 \\
c=-\infty \\
d=0\end{array}$ & $\begin{array}{l}\text { Modelo IV } \\
a=-2,44 \\
b=6,63 \\
c=4,13 \\
d=6,63\end{array}$ & $\begin{array}{l}\text { Modelo I } \\
a=3,23 \\
b=0 \\
c=-\infty \\
d=0\end{array}$ \\
\hline Amphiura crassipes & $\begin{array}{l}\text { Modelo IV } \\
\mathrm{a}=-3,27 \\
\mathrm{~b}=8,98 \\
\mathrm{c}=4,45 \\
\mathrm{~d}=8,98\end{array}$ & $\begin{array}{l}\text { Modelo II } \\
a=4,73 \\
b=-3,48 \\
c=-\infty \\
d=0\end{array}$ & $\begin{array}{l}\text { Modelo IV } \\
\mathrm{a}=-2,42 \\
\mathrm{~b}=7,91 \\
\mathrm{c}=4,34 \\
\mathrm{~d}=7,91\end{array}$ & $\begin{array}{l}\text { Modelo I } \\
a=3,18 \\
b=0 \\
c=-\infty \\
d=0\end{array}$ & $\begin{array}{l}\text { Modelo II } \\
a=2,60 \\
b=4,46 \\
c=-\infty \\
d=0\end{array}$ & $\begin{array}{l}\text { Modelo I } \\
a=3,18 \\
b=0 \\
c=-\infty \\
d=0\end{array}$ \\
\hline Arbacia dufresnei & $\begin{array}{l}\text { Modelo II } \\
a=1,57 \\
b=4,58 \\
c=-\infty \\
d=0\end{array}$ & $\begin{array}{l}\text { Modelo II } \\
a=1,68 \\
b=2,45 \\
c=-\infty \\
d=0\end{array}$ & $\begin{array}{l}\text { Modelo II } \\
a=0,73 \\
b=8,39 \\
c=-\infty \\
d=0\end{array}$ & $\begin{array}{l}\text { Modelo I } \\
a=2,24 \\
b=0 \\
c=-\infty \\
d=0\end{array}$ & $\begin{array}{l}\text { Modelo V } \\
a=1,12 \\
b=2,98 \\
c=1,64 \\
d=135,73\end{array}$ & $\begin{array}{l}\text { Modelo III } \\
a=462,85 \\
b=-1723,84 \\
c=1,95 \\
d=0\end{array}$ \\
\hline $\begin{array}{l}\text { Pseudechinus } \\
\text { magellanicus }\end{array}$ & $\begin{array}{l}\text { Modelo I } \\
a=1,58 \\
b=0 \\
c=-\infty \\
d=0\end{array}$ & $\begin{array}{l}\text { Modelo II } \\
a=0,84 \\
b=3,44 \\
c=-\infty \\
d=0\end{array}$ & $\begin{array}{l}\text { Modelo I } \\
a=1,58 \\
b=0 \\
c=-\infty \\
d=0\end{array}$ & $\begin{array}{l}\text { Modelo II } \\
a=2,18 \\
b=-1,36 \\
c=-\infty \\
d=0\end{array}$ & $\begin{array}{l}\text { Modelo II } \\
a=1,89 \\
b=-1,12 \\
c=-\infty \\
d=0\end{array}$ & $\begin{array}{l}\text { Modelo IV } \\
a=-2,44 \\
b=8,03 \\
c=2,72 \\
d=8,03\end{array}$ \\
\hline Trypilaster philippii & $\begin{array}{l}\text { Modelo IV } \\
a=-9,30 \\
b=11,54 \\
c=8,70 \\
d=11,54\end{array}$ & $\begin{array}{l}\text { Modelo IV } \\
a=-3,83 \\
b=12,25 \\
c=5,13 \\
d=12,25\end{array}$ & $\begin{array}{l}\text { Modelo IV } \\
a=-16,45 \\
b=22,05 \\
c=15,66 \\
d=22,05\end{array}$ & $\begin{array}{l}\text { Modelo II } \\
a=2,73 \\
b=3,32 \\
c=-\infty \\
d=0\end{array}$ & $\begin{array}{l}\text { Modelo II } \\
a=1,29 \\
b=394,21 \\
c=-\infty \\
d=0\end{array}$ & $\begin{array}{l}\text { Modelo V } \\
a=-6,42 \\
b=36,82 \\
c=135,04 \\
d=812,02\end{array}$ \\
\hline
\end{tabular}


El erizo espatangoideo Trypilaster philippii se relaciona con las mayores profundidades muestreadas, sobre las que presenta un óptimo hacia los $135 \mathrm{~m}$ de profundidad; se relaciona asimismo con sustratos con porcentajes altos de la fracción limos-arcillas (óptimo en un $75 \%$ ) y arenas muy finas (óptimo en el $25 \%$ ), es decir con un neto predominio de la primera fracción. La especie presenta un óptimo respecto de las temperaturas de verano en unos $12,1^{\circ} \mathrm{C}$ (modelo unimodal asimétrico), menor que el promedio de éstas (promedio $=12,4^{\circ} \mathrm{C}$ ) y una relación monotónica decreciente con las corrientes de superficie, es decir que su máxima abundancia se encuentra en áreas con corrientes de superficie entre 0 y $0,33 \mathrm{~m} / \mathrm{s}$, estando su promedio ponderado relacionado con corrientes menores que el promedio de las muestras consideradas (promedio = $0,51 \mathrm{~m} / \mathrm{s}$ ). El modelo monotónico decreciente se aplica asimismo a la relación de $T$. philippii con la abundancia de la fracción de arenas muy gruesas, estando la presencia de la especie asociada a porcentajes menores al $1 \%$ de esta fracción.

El óptimo del holoturoideo Chiridota marenzelleri se relaciona con porcentajes mayores que el promedio de las fracciones de limos-arcillas (óptimo en 57\%) y arenas muy finas (óptimo en 33\%). La abundancia de la especie aumenta con la profundidad, estando su promedio ponderado por encima del promedio de las profundidades del muestreo $(58 \mathrm{~m})$. Por otra parte y de acuerdo al ajuste a los modelos HOF, presenta relaciones uniformes con las temperaturas de verano, las corrientes y el porcentaje de arenas muy gruesas.

El ofiuroideo Amphiura crassipes tiene su óptimo a profundidades algo menores a los $80 \mathrm{~m}$ y en sustratos con porcentajes altos de la fracción limos-arcillas (42\%); su abundancia aumenta (relación monotónica creciente) con el aumento del porcentaje de la fracción de arenas muy finas, estando su promedio ponderado relacionado con porcentajes mayores que el promedio (14\%) de esta fracción. La abundancia de $A$. crassipes disminuye con el aumento de la fracción de arenas muy gruesas, estando su promedio ponderado asociado a porcentajes menores que el promedio para esta fracción (9\%). La abundancia de la especie presenta relaciones de tipo uniforme con la temperatura de verano y las corrientes de superficie, o sea que no manifiesta una respuesta a cambios en estas variables.

Las siguientes especies se hallan cerca del origen de coordenadas del CCA y su situación particular es mejor descripta a través de los modelo HOF que con el CCA:

El ofiuroideo Ophiactis asperula no se relaciona con una profundidad en particular, mientras que su abundancia presenta un ajuste monotónico decreciente con las fracciones de limos-arcillas y de arenas muy finas y ajusta a un modelo uniforme respecto de la fracción de arenas muy gruesas. Su abundancia presenta una relación monotónica decreciente con las temperaturas de verano. En cambio, su abundancia aumenta con la velocidad de las corrientes de superficie, estando su promedio ponderado por encima de las corrientes medias $(0,51 \mathrm{~m} / \mathrm{s})$.

El erizo Pseudechinus magellanicus presenta una respuesta uniforme a los cambios de profundidad o del porcentaje de la fracción de limos-arcillas; su abundancia disminuye con el aumento de la fracción de arenas muy finas, aumenta con el aumento de la fracción de arenas muy gruesas y con la velocidad de las corrientes de superficie y presenta un óptimo de temperatura de verano hacia los $12,3^{\circ} \mathrm{C}$ (algo menos del promedio).

El ofiuroideo Amphiura magellanica presenta un óptimo en sustratos con porcentajes del orden del 18\% de arenas muy gruesas (superiores al promedio de la fracción); no se relaciona con una profundidad $\mathrm{o}$ temperatura en particular; su abundancia se relaciona positivamente con el aumento en las corrientes de superficie y negativamente con el aumento del porcentaje de las fracciones de limos-arcillas y arenas muy finas.

La abundancia del erizo Arbacia dufresnei presenta una relación inversa con el aumento de la profundidad y de las fracciones sedimentarias muy finas, presenta un máximo a porcentajes del orden del $2 \%$ de la fracción de arenas muy gruesas (ajustando a un modelo unimodal asimétrico hacia los porcentajes mas altos de la fracción), es indiferente a las corrientes de superficie y ajusta a un modelo monotónico asintótico con las temperaturas de verano, con una asíntota de abundancias altas por encima de $\operatorname{los} 12,3^{\circ} \mathrm{C}$.

El ofiuroideo Ophioplocus januarii presenta su óptimo de abundancia a bajas profundidades $(30 \mathrm{~m})$, en sustratos del orden del promedio de las fracción de arenas muy gruesas y en áreas con temperaturas de verano superiores al promedio (óptimo en unos $12,6^{\circ} \mathrm{C}$ ), se asocia negativamente (función monotónica decreciente) con la fracción de arenas muy finas en tanto que presenta una respuesta uniforme a cambios en la fracción de limos arcillas y corrientes de superficie.

La abundancia del holoturoideo Pentamera chiloensis ajusta a sendos modelo I (uniforme) con la profundidad, tipo de sedimentos (fracciones muy finas o muy gruesas), temperaturas de verano y corrientes de superficie.

\section{Discusión y Conclusiones}

Los patrones de distribución dentro de los golfos, obtenidos con el método de "point kriging" de todas 
las especies de equinodermos estudiadas, indican ausencia o presencia en bajas densidades de las mismas en la mitad oriental del golfo San José. Este sector presenta temperaturas extremas tanto en verano como en invierno y dominancia de sustratos de granulometría muy fina.

Los rangos de distribución vertical de las especies coinciden en general con los indicados por Bernasconi \& D'Agostino (1977) y Bartsch (1982), pero los de Amphiura crassipes son mayores que los indicados por Bernasconi \& D'Agostino (1977) para el Mar Argentino, alcanzando la especie los $120 \mathrm{~m}$ de profundidad en el golfo San Matías.

La hipótesis de la exclusión trófica mutua propone que la composición, biomasa y productividad de la comunidades de la macrofauna bentónica están limitadas por el alimento y que el mecanismo básico de exclusión entre suspensívoros y detritívoros es la velocidad de la corriente de agua sobre el sedimento. Las asociaciones de detritívoros están relacionadas con corrientes bajas y sedimentos con un diámetro de partículas menor de $63 \mu \mathrm{m}$, sujetos a una intensa reelaboración biológica. Las asociaciones de suspensívoros en cambio, se desarrollan en áreas con corrientes de alta velocidad y, al menos en aquellas que se desarrollan en sedimentos blandos, involucran algún medio de estabilización de la interfase agua-sedimento (Wildish 1977). En las primeras, la exclusión de los suspensívoros tiene lugar por el aumento de la turbidez y la inestabilidad del sedimento; en las segundas la exclusión de los detritívoros se lleva a cabo por la eliminación de los detritos resuspendidos que constituyen su fuente de alimento. En la siguiente discusión se asume que la distribución de las especies consideradas se halla en función de las condiciones hidrográficas, las cuales permiten algunos tipos de alimentación y excluyen a otros (Wildish 1977) y condicionan asimismo el tipo de sedimento dominante (Riedl 1971).

Los erizos espatangoideos, a los que pertenece Trypilaster philippii, son considerados limnívoros no selectivos, es decir que no llevan a cabo una selección del alimento en los sedimentos que ingieren (Turpaeva 1953, según Pérès 1982a, Sokolova 1959, según Pérès 1982a, Hammond 1980); la asociación del óptimo de la especie con porcentajes del $75 \%$ de la fracción limosarcillas y su tubo digestivo lleno de barro, parecen sustentar la presunción de un comportamiento trófico semejante. Los estudios llevados a cabo sobre integrantes del orden, señalan que los mismos llevan a cabo la explotación de bacterias y quizás detritos del sedimento como fuente de carbono orgánico (Hammond 1980). Algunas especies como Brissopsis lyrifera son consideradas como consumidoras sub-superficiales someras en barros con alto contenido orgánico (Buchanan 1967), en tanto que otras como Echinocardium cordatum son consideradas consumidores de depósito facultativos, pudiendo cambiar de colectores de superficie a consumidores de subsuperficie de acuerdo a los ambientes (Buchanan 1966); esta última especie puede utilizar la chimenea en forma de embudo de su galería como trampa selectiva, llegando el sedimento atrapado a ser unas cuatro veces más rico en materia orgánica que el sedimento de superficie (De Ridder \& Jangoux 1987). Las preferencias de Trypilaster philipii por sustratos con dominancia de limos y arcillas, son semejantes a las de las especies del género Brissopsis (Buchanan 1967, Pérès 1982b). A pesar de la existencia de sustratos con dominancia de esta granulometría en grandes áreas del golfo san José, la especie se halla excluida del mismo; es probable que los sedimentos finos del golfo San José, dada la relativamente baja profundidad de este golfo, no sean lo suficientemente estables como para permitir su establecimiento; otra razón podrían ser las temperaturas de verano en la zona donde estos sedimentos se encuentran. La relación inversa de la abundancia de la especie con las corrientes de superficie está en relación con la correlación negativa existente entre la abundancia de la fracción limos-arcillas con la que la especie se halla asociada y la velocidad de las corrientes.

El holoturoideo sináptido Chiridota marenzelleri puede ser considerado, a partir de las preferencias de otros integrantes del grupo, como limnívoro no selectivo (Turpaeva 1953, según Pérès, 1982a, Sokolova 1959, según Pérès 1982a); un comportamiento trófico semejante puede inferirse a partir de la asociación de la especie con sustratos de granulometría muy fina, con óptimos de abundancia a porcentajes altos de estas fracciones. La distribución y abundancia de la especie Chiridota rigida y de otros chiridótidos se halla condicionada por el sustrato en el cual excavan; la habilidad para excavar aumenta con la proporción de partículas finas presentes en el sedimento y con el aumento en la clasificación de éstos (Lawrence \& Murdoch 1977).

Tanto Trypilaster philippii como Chiridota marenzelleri forman parte de la comunidad de Eunice frauenfeldi - Trypilaster - Chiridotidae, descripta para el golfo San Matías en la cuenca central fangosa ubicada por debajo de la isobata de los $70 \mathrm{~m}$ (Escofet et al. 1978) o de la asociación de Chiridota marenzelleriPeltarium spinosulum descripta para la zona sur del mismo golfo y relacionada con profundidades mayores de $100 \mathrm{~m}$ y sustratos con predominio de limos-arcillas y porcentajes medios de arenas muy finas (Zaixso et al. 1998). De acuerdo a Carriquiriborde et al. (1983), la presencia de Trypilaster philippii en el golfo Nuevo, se extiende a sustratos arcillo-limosos, limo-arenosos y 
arenosos.

El patrón de distribución de Pentamera chiloensis y la ausencia de relaciones de esta especie con los parámetros ambientales seleccionados por el CCA, no ayuda a dilucidar su modo de vida. Se considera a los dendrochirótidos tanto consumidores de depósitos como filtradores pasivos; los podia bucales ramificados son utilizados para la captura o la toma de partículas que luego son llevadas a la boca (Pandian 1975, Smith 1983). El patrón de distribución de la especie, una serie de núcleos dispersos, es semejante al observado para Cucumaria elongata en las comunidades bentónicas costeras de fondos areno-limosos de Northumberland (Buchanan 1967); esta especie es considerada como consumidora de depósitos con la capacidad de seleccionar partículas por su tamaño (Pandian 1975). Pentamera chiloensis es especie preferente de la asociación de los golfos San José - San Matías caracterizada por Chiridota marenzelleri - Peltarium spinosulum (Zaixso et al. 1998).

Se considera que los ofiuroideos de la familia Amphiuridae pueden presentar diferentes modos de obtención de alimento que van desde la alimentación suspensívora hasta la colección de partículas alimenticias de la capa de sedimento superficial (Turpaeva 1953, según Pérès 1982a, Sokolova 1959, según Pérès 1982a, Buchanan 1964).

La relación de la especie Amphiura crassipes con sedimentos muy finos y sitios profundos podría indicar un modo de alimentación relacionado con la obtención de partículas alimenticias de la superficie de los sedimentos. En apoyo a esta suposición se puede señalar que la especie $A$. chiajei, considerada como recolector de superficie por Buchanan (1964) se asocia con sedimentos fangosos profundos en algunos loch escoceses y rías gallegas (Pearson 1970, López Jamar 1981). Amphiura crassipes es especie integrante de las asociaciones de fondos de granulometría muy fina (B y C) de los golfos San José - San Matías caracterizadas por especies de poliquetos (Zaixso et al. 1998).

La distribución del ofiuroideo Amphiura magellanica y su asociación con sustratos de granulometría gruesa y corrientes fuertes, podría implicar un modo de alimentación suspensívoro pasivo.

El modo de alimentación de algunas especies del género Ophiactis ha sido caracterizado como suspensívoro pasivo (Lee et al. 1983, Giacobbe \& Rinelli 1992). La asociación de la especie Ophiactis asperula con fuertes corrientes (particularmente en la zona de la desembocadura del golfo San José) permiten suponer un modo de alimentación semejante; resulta interesante remarcar que si bien la abundancia de la especie se halla en relación inversa con el aumento de las fracciones sedimentarias más finas, la misma presenta una respuesta uniforme a cambios en el porcentaje de las arenas muy gruesas. La especie Ophiactis balli se distribuye en el estrecho de Messina en poblaciones densas sobre fondos duros circalitorales caracterizados por fuertes corrientes de marea (Giacobbe \& Rinelli 1992). Por otra parte, la ubicación de $O$. asperula en el talud de la costa sur del golfo San Matías, se asemeja a la distribución de Amphiura filiformis, en el estrecho de Skagerrak donde la especie en cuestión es dominante en los sitios profundos donde la disminución de las fuertes corrientes de fondo existentes permite la sedimentación de partículas orgánicas e inorgánicas transportadas (Rosemberg 1995). La especie Amphiura filiformis es considerada tanto una especie suspensívora como una consumidora de depósitos de superficie (Creutzberg et al. 1984). La ausencia de respuesta de Ophiactis asperula a los cambios de profundidad como resulta de los modelos HOF, coincide con que la mayor parte de los hallazgos de esta especie en la plataforma argentina fueron encontrados entre los 0 a $300 \mathrm{~m}$ de profundidad, si bien algunos ejemplares fueron hallados a $1000 \mathrm{~m}$ (Bartsch 1982).

Es poco lo que se puede indicar de Ophioplocus januarii, excepto que la abundancia de la especie presenta un óptimo en aguas someras, con porcentajes del orden de la media de la fracción de arenas muy gruesas y con temperaturas de verano altas. En el golfo San José este tipo de ambientes está caracterizado por comunidades dominadas por suspensívoros (Zaixso 1997); sin embargo la falta de relación de la especie con las corrientes, su abundancia relativamente escasa (comparada con especies caracterizadas como filtradoras pasivas) y su gran tamaño sugieren más bien un modo de alimentación de tipo oportunista y aún la predación circunstancial. De acuerdo a Tommasi (1962, 1970, según Bartsch 1982) la especie se encuentra tanto en sustratos duros como blandos ricos en detritos. Bartsch (1982) señala que los pocos especímenes disecados, presentaban sus estómagos vacíos a excepción de granos de sedimento.

Las especies Amphiura magellanica, Ophiactis asperula y Ophioplocus januarii son integrantes de la asociación de supensívoros-raspadores de los golfos San José-San Matías (asociación D), caracterizada entre otras especies por el mitílido Aulacomya atra atra; Ophiactis asperula es característica de la asociación, en tanto que Ophioplocus januarii es una especie preferencial de la misma (Zaixso et al. 1998).

El erizo Arbacia dufresnei se halla asociado a zonas poco profundas, a la ausencia de fracciones sedimentarias finas $y$ un promedio ponderado relacionado con porcentajes medios de arenas muy 
gruesas y relacionado con un modelo unimodal asimétrico hacia los porcentajes más altos de esta fracción; la primera de las asociaciones posiblemente responda también por la relación de Arbacia con áreas con temperaturas de verano altas. Si bien la presencia de toba no fue seleccionada como variable por el CCA, se debe señalar que este tipo de fondos está fuertemente relacionado con los fondos con predominio de fracciones muy gruesas. La asociación de Arbacia con sedimentos gruesos (arenas) y sustratos duros ha sido indicada previamente para el golfo Nuevo (Carriquiriborde et al. 1983) y para el golfo San José (Zaixso et al., 1998), donde caracteriza (en parte) a la asociación de raspadores-supensívoros. Varias especies del género han sido señaladas como consumidoras de macroalgas (Hay et al. 1986, Klinger \& Lawrence 1985, Marques 1984) y son constituyentes comunes de asociaciones infralitorales con predominio de algas fotófilas blandas o de sus facies (Pérès 1982b); si bien en el CCA la presencia de algas no resultó ser una variable significativa, la asociación de $A$. dufresnei con bajas profundidades sugiere una asociación semejante. Algunos autores consideran a las especies de Arbacia como predadoras, ya que denudan el sustrato de casi todas las especies que en él se encuentran (Karlson 1978, Bergin 1987). Penchaszadeh (1979) señala que la dieta de $A$. dufresnei, en bancos circalitorales de mejillones de la costa argentina Norte, se halla integrada por cirripedios y poliquetos (los componentes más constantes a lo largo del año), huevos y juveniles (generalmente menores de $1 \mathrm{~mm}$ ) de mejillones (estos dos últimos ítems durante la freza y el reclutamiento de la especie), otros bivalvos y gasterópodos pequeños, diversos crustáceos (cumáceos, ostrácodos y anfípodos) y foraminíferos.

El erizo Pseudechinus magellanicus presenta una amplia distribución dentro del área estudiada y parece estar relacionado principalmente con sitios con corrientes de superficie fuertes, dominados por sustratos gruesos y profundidades intermedias. Esta distribución señalaría una forma de alimentación diferente a la de Arbacia y que podría estar relacionada al consumo tanto de alimento vivo (algas y animales) como de detritos aportados por las corrientes. La especie ha sido señalada como herbívora en el bentos asociado a bosques de Macrocystis pyrifera del canal de Beagle (Ojeda \& Santelices 1984; Adami \& Gordillo 1999) y como consumidora secundaria en bancos circalitorales de mejillón, alimentándose de cirripedios, poliquetos, reclutas de bivalvos, pequeños gasterópodos, crustáceos, foraminíferos y restos de crustáceos diversos (Penchaszadeh 1979). Pseudechinus magellanicus ha sido indicada como integrante de cuatro grandes asociaciones del macrozoobentos del golfo San José (Zaixso et al. 1998), lo que refleja su amplia distribución en el golfo. La distribución de la especie en el golfo Nuevo es asimismo amplia, como se desprende de su pertenencia a diferentes comunidades del golfo (Carriquiriborde et al. 1983).

De las anteriores observaciones, resulta que tanto Arbacia dufresnei como Pseudechinus magellanicus son especies oportunistas en cuanto a su alimentación, lo que explicaría en parte su amplia distribución en la zona de estudio.

Se concluye que en el golfo San José y sur del golfo San Matías es posible señalar:

1. Un conjunto de especies formado por los erizos Arbacia dufresnei y Pseudechinus magellanicus y los ofiuroideos Ophioplocus januarii, Amphiura magellanica y Ophiactis asperula que presentan requerimientos ambientales aproximadamente semejantes; en particular se puede indicar que todas ellas presentan relaciones monotónicas negativas con el aumento de la fracción de arenas muy finas y se asocian positivamente con corrientes de superficie fuertes (Pseudechinus magellanicus, Amphiura magellanica y Ophiactis asperula); si son indiferentes a este último factor se asocian entonces a ambientes poco profundos (Arbacia dufresnei y Ophioplocus januarii). Este conjunto se asocia tanto a una alimentación con búsqueda activa de alimento (A. dufresnei, P. magellanicus y posiblemente $O$. januarii), como a una probable alimentación por aporte de detritos gruesos o filtración pasiva ( $A$. magellanica y $O$. asperula). Ophiactis asperula y Pseudechinus magellanicus son las especies de equinodermos más abundantes en el área de estudio.

El holoturoideo Pentamera chiloensis si bien cercano al grupo anterior, no se relaciona con ninguno de los parámetros ambientales analizados.

2. Las restantes especies analizadas (Trypilaster philippii, Amphiura crassipes y Chiridota marenzelleri), se caracterizan por habitar ambientes con características diferenciales. Todas ellas tienen en común su preferencia por sustratos muy finos, los cuales se asocian por lo general a un modo de alimentación detritívoro o limnívoro.

Las formas de alimentación postuladas en este trabajo en base a las afinidades taxonómicas de las especies y a sus relaciones ambientales son especulativas y requieren confirmación, siendo esta necesidad de profundización del conocimiento sobre la autoecología, común a un gran número de especies bentónicas de la costa argentina. 


\section{Agradecimientos}

Se agradece al Servicio Centralizado de Química del Centro Nacional Patagónico los datos referidos a los parámetros del agua de mar del golfo San José que han sido utilizados en este trabajo. Se agradece asimismo a los dos revisores anónimos del manuscrito, cuyas valiosas sugerencias contribuyeron a mejorar este trabajo.

\section{Literatura Citada}

Adami ML \& S Gordillo. 1999. Structure and dynamics of the biota associated with Macrocystis pyrifera (Phaeophyta) from the Beagle Channel, Tierra del Fuego. Scientia Marina 63 (Supl. 1): 183-191.

Anderson DA. 1988. Some models for overdispersed binomial data. Australian Journal of Statistics 30: 125-148.

Bartsch I. 1982. Ophiuroidea (Echinodermata) from the Patagonian Shelf. Mitteilungen Hamburgisches Zoologisches Museum und Institut 79: 211-250.

Bastida R, A Roux \& D Martínez. 1992. Benthic communities of the Argentine continental shelf. Oceanologica Acta 15 (6): 687-698.

Bergin F. 1987. Contenus digestifs de Paracentrotus lividus et d'Arbacia dans la region d'El Dabaa (Egypte). En: Boudouresque CF (ed), International Colloquium on Paracentrotus lividus and edible sea-urchins, France: 107116.

Bernasconi I \& MM D’Agostino. 1977. Ofiuroideos del Mar epicontinental Argentino. Revista del Museo Argentino de Ciencias Naturales, Hidrobiología, Buenos Aires 5 (5): 65-125.

Boraso de Zaixso AL, HE Zaixso \& GN Casas. 1999. Asociaciones de algas bentónicas submareales del golfo San José (Chubut, Argentina). Physis (Buenos Aires) Secc. A 57 (132-133): 17-27.

Buchanan JB. 1964. A comparative study of some features of the biology of Amphiura filiformis and Amphiura chiajei (Ophiuroidea) considered in relation to their distribution. Journal of the Marine Biological Association of the United Kingdom 44: 565-576.

Buchanan JB. 1966. The biology of Echinocardium cordatum (Echinodermata: Spatangoidea) from different habitats. Journal of the Marine Biological Association of the United Kingdom 46: 97-114.

Buchanan JB. 1967. Dispersion and demography of some infaunal echinoderm populations. En: N Millot (ed) Echinoderm biology, Symposia of the Zoological Society of London 20: 1-11. Academic Press.

Carriquiriborde L, C Borzone, $Z$ Lizarralde, A Pombo, R Manrique \& M Ichazo. 1983. Aspectos biocenológicos del golfo Nuevo (Chubut, Argentina), 12 p. Centro Nacional Patagónico, Informe Interno, Puerto Madryn, Argentina.
Creutzberg F, P Wapenar, G Duineveld \& N López López. 1984. Distribution and density of the benthic fauna in the southern North Sea in relation to bottom characteristics and hydrographic conditions. Rapports et Process-verbaux des Réunions du Conseil Permanent International pour l'Exploration de la Mer 183: 101-110.

De Ridder C \& M Jangoux. 1987. Comportement alimentaire de l'oursin spatangidae Echinocardium cordatum. En: C F Boudouresque (ed) International Colloquium on Paracentrotus lividus and edible seaurchins, France: 139-144.

Escofet A, JM Orensanz, SR Olivier \& V Scarabino. 1978. Biocenología bentónica del golfo San Matías (Río Negro, Argentina): Metodología, experiencias y resultados del estudio ecológico de un gran espacio geográfico en América Latina. Anales Centro Ciencias del Mar y Limnología, Universidad Nacional Autónoma de México 5 (1): $59-82$.

Giacobbe S \& P Rinelli. 1992. Ecological notes on Ophiactis balli (Thomson) from populations of Errina aspera in the Straits of Messina. En: Scalera Liaci L \& C Canicatti (eds), Echinoderm Research: 202. Rotterdam, Netherlands, Balkema.

Hammond LS. 1980. Diversity and coexistence in an assemblage of deposit-feeding holothurians and spatangoids of the reef lagoon, Discovery Bay, Jamaica. Proceedings of the Association of Island Marine Laboratories of the Caribbean 15: 11.

Hay ME, RR Lee, RA Guieb \& MM Bennett. 1986. Food preference and chemotaxis in the sea urchin Arbacia punctulata (Lamarck) Philippi. Journal of Experimental Marine Biology and Ecology 96 (2): 147-153.

Huisman J, H Olff \& LFM Fresco. 1993. A hierarchical set of models for species response analysis. Journal of Vegetation Science 4: 37-46.

Ingram P. 1995. SSTAT. Version 3.01 (Spatial and Map Analysis). User Manual. Disponible en: http://atlas.es.mq.edu.au/users/pingram/sware.htm, 106 pp $(15 / 10 / 1998)$.

Isaaks EH \& RM Srivastava. 1989. Applied geostatistics, 561 p. Oxford University Press, New York.

Karlson R. 1978. Predation and space utilization patterns in marine epifaunal community. Journal of Experimental Marine Biology and Ecology 31: 225-239.

Klinger TS \& JM Lawrence. 1985. The hardness of the teeth of five species of echinoids (Echinodermata). Journal of Natural History 19: 917-920.

Lawrence JM \& J Murdoch. 1977. The effect of particle size frequency distribution of the substratum on the burrowing ability of Chiridota rigida (Semper) (Echinodermata: Holothuroidea). Marine and Behaviour Physiology 4: 305-311.

Lee JH, JS Hong \& SK Yi, 1983. Studies on the benthic fauna in Garolin Bay, Korea. Subtidal soft-bottom community. Journal of the Oceanological Society of Korea 18: 111-116. 
López Jamar E. 1981. Spatial distribution of the infaunal benthic communities of the ria de Muros, North-West Spain. Marine Biology 63: 29-27.

Marques VM. 1984. Effects of Arbacia lixula (L.) (Echinoidea) on the algal communities of Sao Miguel and Graciosa Islands (Azores Archipielago, Portugal). Arquivos do Museu Bocage, Serie B, Notas e Suplementos 2: 103-108.

Ojeda FP \& B Santelices. 1984. Invertebrate communities in holdfast of the kelp Macrocystis pyrifera from southern Chile. Marine Ecology Progress Series 16 (1-2): 65-73.

Oksanen J. 1996. HOF: Gradient analysis using HuismanOlff-Fresco models with maximun likelihood. Disponible en: http://www.helsinki.fi/ jhoksane/pages/hof $2 . h t m l$ $(6 / 12 / 1998)$.

Oksanen J. 1998. HOF: Ecological gradient analysis using Huisman-Olff-Fresco models. Version 2.3. Disponible en: http://www.helsinki.fi/ jhoksane/softhelp/hofread.html $(17 / 10 / 1999)$

Palma ED \& DD Serman. 1984. Tidal energy in Valdes Peninsula. Consejo Nacional de Investigaciones Científicas y Técnicas, Informe no publicado, $32 \mathrm{p}$. Buenos Aires, Argentina.

Pandian TJ. 1975. Mechanisms of heterotrophy. En: O Kinne (ed), Marine Ecology 2 (1): 61-249. John Wiley \& Sons Ltd..

Pearson TH. 1970. The benthic ecology of Loch Linnhe and Loch Eil, a sea-loch system on the West coast of Scotland. 1. The physical environment and distribution of the macrobenthic fauna. Journal of Experimental Marine Biology and Ecology 5: 1-34.

Penchaszadeh P. 1979. Estructura de la comunidad y procesos que la determinan en bancos circalitorales de mejillón Mytilus platensis. Memorias del Seminario sobre ecología bentónica y sedimentación de la plataforma continental del Atlántico Sur. UNESCO, Montevideo: 131-145.

Pérès JM. 1982a. Structure and dynamics of assemblages in the benthal. En: O Kinne (ed), Marine ecology 5 (1): 119185. John Wiley \& Sons Ltd.

Pérès JM. 1982b. Major benthic assemblages. En: O Kinne (ed), Marine ecology 5 (1): 373-522. John Wiley \& Sons Ltd.

Riedl R. 1971. Water movement. Animals. En: O Kinne (ed), Marine ecology 1 (2): 1123-1156. John Wiley \& Sons Ltd.
Rosemberg R. 1995. Benthic marine fauna structured by hydrodinamic processes and food avalaibility. Netherland Journal of Sea Research 34: 303-317.

Smith TB. 1983. Tentacular structure and feeding behaviour of Neopentadactyla mixta (Holothuroidea: Dendrochirota). Journal of the Marine Biological Association of the United Kingdom 63: 301-311.

ter Braak CJF. 1987. CANOCO. A FORTRAN program for canonical community ordination by [partial] [detrended] [canonical] correspondence analysis, principal components analysis and redundancy analysis (version 2.1), 95 p. TNO Institute for Applied Computer Science, Statistics Department Wageningen, 6700 AC Wageningen, The Netherlands.

ter Braak CJF. 1990. Update notes. CANOCO version 3.10, 35 p. Agricultural Mathematics Group, Wageningen, The Netherlands.

ter Braak CJF. 1995. Ordination. En: Jongman R, C ter Braak. \& O van Tongeren (eds), Data analysis in community and landscape ecology (Second Edition): 91173. Cambridge University Press, Cambridge.

Wildish DJ. 1977. Factors controlling marine and estuarine sublittoral macrofauna. Helgoländer wiss. Meeresunters., 30: $445-454$

Zaixso HE. 1996a. Distribución y abundancia de bivalvos de fondos blandos submareales del golfo San José y sur del golfo San Matías (Chubut, Argentina). Medio Ambiente (Chile) 13 (1): 97-113

Zaixso HE. 1996b. Distribución de Chlamys tehuelcha y Chlamys patagonica (Bivalvia, Pectinidae) en el golfo San José (Chubut, Argentina) en función de la profundidad y el sustrato. Physis (Buenos Aires) Sección A 51 (120): 1-11.

Zaixso HE. 1997. Asociaciones de moluscos bentónicos submareales de moluscos del golfo San José y sur del golfo San Matías (Chubut, Argentina). Physis (Buenos Aires) Sección A 54 (126-127): 1-21.

Zaixso HE. 1999. Distribución submareal del mitílido Aulacomya atra atra (Molina) en el golfo San José (Argentina) en relación con la profundidad, características del sustrato y condiciones hidrográficas. Physis (Buenos Aires) Secc. A 57 (132-133): 1-10.

Zaixso HE, CT Pastor, ZI Lizarralde, E Gómes Simes, E Romanello \& G Pagnoni. 1998. Macrozoobentos del golfo San José. Revista Biología Marina y Oceanografía 33 (1): 43-72. 